\title{
Managing Risk Exposures using the Risk Budgeting Approach*
}

\author{
Benjamin Bruder \\ Research \& Development \\ Lyxor Asset Management, Paris \\ benjamin. bruder@lyxor.com \\ Thierry Roncalli \\ Research \& Development \\ Lyxor Asset Management, Paris \\ thierry.roncalli@lyxor.com \\ First version: January 2012 - This version: March 2012
}

\begin{abstract}
The ongoing economic crisis has profoundly changed the industry of asset management by putting risk management at the heart of most investment processes. This new risk-based investment style does not rely on return forecasts and is therefore assumed to be more robust. 2011 was marked by several great successes in transforming the practice of asset management with several large institutional investors moving their portfolios to minimum variance, ERC or risk parity strategies. These portfolio constructions are special cases of a more general class of allocation models, known as the risk budgeting approach. In a risk budgeting portfolio, the risk contribution from each component is equal to the budget of risk defined by the portfolio manager. Unfortunately, even if risk budgeting techniques are widely used by market practitioners, there are few results about the behavior of such portfolios in the academic literature. In this paper, we derive the theoretical properties of the risk budgeting portfolio and show that its volatility is located between those of the minimum variance and weight budgeting portfolios. We also discuss the existence, uniqueness and optimality of such a portfolio. In the second part of the paper, we propose several applications of risk budgeting techniques for risk-based allocation, like risk parity funds and strategic asset allocation, and equity and bond alternative indexations.
\end{abstract}

Keywords: risk budgeting, risk management, risk-based allocation, equal risk contribution, diversification, concentration, risk parity, alternative indexation, strategic asset allocation.

JEL classification: G11, C58, C60.

\section{Introduction}

The investment industry of developed countries has changed with the subprime crisis of 2008 and the ongoing crisis of sovereign debt. For example, the number of mutual funds in Europe has decreased by 5\% since 2008 after a growth of $25 \%$ between 2004 and 2008 (Investment Company Institute, 2011). Retail investors' risk aversion is ever increasing and they have turned to asset managers who had achieved to manage the financial crisis in a robust way. In the mean time, the business of hedge funds has evolved completely. It is now driven by more transparent investment vehicles, like newcits funds and managed accounts,

\footnotetext{
${ }^{*}$ We thank seminar participants at ESSEC Business School (WG Risk) and Guillaume Weisang for their helpful comments.
} 
and is dominated by large fund managers. Investment management for institutional clients faces the same issues:

"With strong capacities in transparency and risk management becoming just as important as overall performance, many institutional investors - insurers, pension funds, corporations, governments and other entities - have put asset managers under scrutiny" (Boston Consulting Group, 2011).

The pressure for more transparency and robustness has therefore modified the relationships between investors and portfolio managers. The time is over when a fund manager could promise the moon. Today, the job of a fund manager is first of all to manage risk.

With the lower risk tolerance of investors, a new risk-based investment style has emerged since the subprime crisis. Although the importance of risk considerations in asset allocation is widely admitted, the idea is often simplified to volatility minimization as described in classical portfolio theory. Mean-variance optimization, however, generally leads to portfolios concentrated in terms of weights. Slight differences in inputs can lead to dramatic changes in allocations and create portfolios heavily invested on very few assets. There is also confusion between optimizing the volatility and optimizing the risk diversification that could be naively described by the general "don't put all your eggs in one basket" concept. It is not the first time that mean-variance portfolio optimization encounters criticisms from investors. In the nineties, academics have proposed to improve the Markowitz model by correcting some drawbacks of this model, such as portfolio resampling (Michaud, 1989) or robust allocation (Tütüncü and Koenig [2004]). But they have continued to keep the same framework, that is minimizing the volatility of the portfolio and targeting an expected return. What is new today is that some investors don't use optimization methods and may prefer some heuristic solutions based on risk concentration.

This new risk-based investment style puts diversification at the heart of the investment process and corresponding strategies share the property that they don't use any performance forecasts as inputs of the model. In a risk budgeting approach, the investor only chooses the risk repartition between assets of the portfolio, without any consideration of returns. In the equal risk contribution (ERC) portfolio, the risk contribution from each portfolio asset is made equal. This portfolio has been extensively studied by Maillard et al. (2010) who had derived several interesting properties. In particular, they have shown that this portfolio is located between minimum variance and equally-weighted portfolios. In some cases, the investor doesn't want to manage the risk exposures in a uniform way. Risk budgets are then not equal. For example, a long-term institutional investor could invest a small part of his strategic portfolio in alternative investments like commodities ${ }^{1}$. Bruder et al. (2011) present also a method to manage sovereign bond portfolios when the risk budget for each country is proportional to the GDP of the country. Unfortunately, even if risk budgeting techniques are widely used by market practitioners, they are few results about the behavior of such portfolios in the academic literature. The goal of this paper is to fill the gap between practice and theory and to give some insights about the behavior of such techniques.

The paper is organized as follows. In section 2, we derive the theoretical properties of the risk budgeting portfolio. We show that its volatility is located between those of minimum variance and weight budgeting portfolios. We also discuss the existence and uniqueness of such a portfolio. By studying the optimality property, we obtain a simple relationship

\footnotetext{
${ }^{1}$ The risk budget of these alternative investments could then represent $5 \%$ or $10 \%$ of the total risk of the portfolio.
} 
between risk contributions and performance contributions. In section 3, we propose several applications of risk budgeting techniques for risk-based allocation, like risk parity funds and strategic asset allocation, and equity and bond alternative indexations. Section 4 draws some conclusions.

\section{Analysis of the risk budgeting approach}

\subsection{Definition}

Let us consider a portfolio of $n$ assets. We define $x_{i}$ as the exposure (or weight) of the $i^{\text {th }}$ asset and $\mathcal{R}\left(x_{1}, \ldots, x_{n}\right)$ as a risk measure for the portfolio $x=\left(x_{1}, \ldots, x_{n}\right)$. If the risk measure is coherent and convex (Artzner et al., 1999), it verifies the following Euler decomposition:

$$
\mathcal{R}\left(x_{1}, \ldots, x_{n}\right)=\sum_{i=1}^{n} x_{i} \cdot \frac{\partial \mathcal{R}\left(x_{1}, \ldots, x_{n}\right)}{\partial x_{i}}
$$

The risk measure is then the sum of the product of the exposure by its marginal risk. In this case, it is natural to define the risk contribution of the $i^{\text {th }}$ asset as follows:

$$
\mathrm{RC}_{i}\left(x_{1}, \ldots, x_{n}\right)=x_{i} \cdot \frac{\partial \mathcal{R}\left(x_{1}, \ldots, x_{n}\right)}{\partial x_{i}}
$$

We consider a set of given risk budgets $\left\{b_{1}, \ldots, b_{n}\right\}$. Here, $b_{i}$ is an amount of risk measured in dollars. The risk budgeting portfolio is then defined by the following constraints:

$$
\left\{\begin{array}{c}
\mathrm{RC}_{1}\left(x_{1}, \ldots, x_{n}\right)=b_{1} \\
\vdots \\
\mathrm{RC}_{i}\left(x_{1}, \ldots, x_{n}\right)=b_{i} \\
\vdots \\
\mathrm{RC}_{n}\left(x_{1}, \ldots, x_{n}\right)=b_{n}
\end{array}\right.
$$

It is also the portfolio such that the risk contributions match the risk budgets. Contrary to the weight budgeting portfolio ${ }^{2}$, we have to solve the system (1) of nonlinear equations to define the risk budgeting portfolio.

In this paper, we focus on the volatility risk measure ${ }^{3}$. We obtain:

$$
\begin{aligned}
\mathcal{R}(x) & =\sigma(x) \\
& =\sqrt{x^{\top} \Sigma x}
\end{aligned}
$$

${ }^{2}$ We note $x_{\mathrm{wb}}$ the weight budgeting portfolio defined by:

$$
x_{i}=b_{i}
$$

${ }^{3}$ We notice however that most of the results could be generalized to other risk measures (see paragraph 2.4 page 13$)$. 
It comes that the marginal risk and the risk contribution of the $i^{\text {th }}$ asset are respectively ${ }^{4}$ :

$$
\begin{aligned}
\frac{\partial \mathcal{R}(x)}{\partial x_{i}} & =\frac{(\Sigma x)_{i}}{\sqrt{x^{\top} \Sigma x}} \\
\mathrm{RC}_{i}\left(x_{1}, \ldots, x_{n}\right) & =x_{i} \cdot \frac{(\Sigma x)_{i}}{\sqrt{x^{\top} \Sigma x}}
\end{aligned}
$$

We check that the volatility verifies the Euler decomposition:

$$
\begin{aligned}
\sum_{i=1}^{n} \mathrm{RC}_{i}\left(x_{1}, \ldots, x_{n}\right) & =\sum_{i=1}^{n} x_{i} \cdot \frac{(\Sigma x)_{i}}{\sqrt{x^{\top} \Sigma x}} \\
& =x^{\top} \frac{\Sigma x}{\sqrt{x^{\top} \Sigma x}} \\
& =\sqrt{x^{\top} \Sigma x} \\
& =\sigma(x)
\end{aligned}
$$

Remark 1 We notice that if the asset returns are Gaussian, the value-at-risk of the portfolio is:

$$
\operatorname{VaR}(x ; \alpha)=\Phi^{-1}(\alpha) \sqrt{x^{\top} \Sigma x}
$$

whereas the expression of the expected shortfall risk measure is:

$$
\operatorname{ES}(x ; \alpha)=\frac{1}{(1-\alpha)} \sqrt{\frac{x^{\top} \Sigma x}{2 \pi}} e^{-\frac{1}{2}\left(\Phi^{-1}(\alpha)\right)^{2}}
$$

Managing risk exposures with value-at-risk or expected shortfall risk measures is then equivalent to manage risk exposures with the volatility in a Gaussian world ${ }^{5}$.

\subsection{The right specification of the $\mathrm{RB}$ portfolios}

The system (1) is too large to define a portfolio which may be interesting to analyse in an asset management point of view. First, it is difficult to think in terms of exposures and nominal risk budgets. That's why we prefer to define the portfolio in terms of weights and the risk budgets in relative value ${ }^{6}$. Second, risk budgeting techniques are used to build diversified portfolios, and specifying that some assets have a negative risk contribution implies that the risk is highly concentrated in the other assets of the portfolio. We also prefer to obtain a long-only portfolio meaning that all the weights are positive. That's why we specify the following mathematical system to define a proper RB portfolio:

$$
\left\{\begin{array}{l}
x_{i} \cdot(\Sigma x)_{i}=b_{i} \cdot\left(x^{\top} \Sigma x\right) \\
b_{i} \geq 0 \\
x_{i} \geq 0 \\
\sum_{i=1}^{n} b_{i}=1 \\
\sum_{i=1}^{n} x_{i}=1
\end{array}\right.
$$

\footnotetext{
${ }^{4}$ Because the vectorial derivative is:

$$
\frac{\partial \mathcal{R}(x)}{\partial x}=\frac{\Sigma x}{\sqrt{x^{\top} \Sigma x}}
$$

${ }^{5}$ Under the assumption that returns are Gaussian, this result is verified for all risk measures that will not depend on the mean of the distribution.

${ }^{6}$ It implies that two constraints $\sum_{i=1}^{n} x_{i}=1$ and $\sum_{i=1}^{n} b_{i}=1$ have to been added to the specification of the problem.
} 
Another difficulty may appear when one specifies that some risk budgets are equal to zero. Let $\Sigma$ be the covariance matrix specified as follows:

$$
(\Sigma)_{i, j}=\rho_{i, j} \sigma_{i} \sigma_{j}
$$

where $\sigma_{i}>0$ is the volatility of the asset $i$ and $\rho_{i, j}$ is the cross-correlation ${ }^{7}$ between the assets $i$ and $j$. It comes that:

$$
\frac{\partial \sigma(x)}{\partial x_{i}}=\frac{x_{i} \sigma_{i}^{2}+\sigma_{i} \sum_{j \neq i} x_{j} \rho_{i, j} \sigma_{j}}{\sigma(x)}
$$

Suppose that the risk budget $b_{k}$ is equal to zero. It means that:

$$
x_{k}\left(x_{k} \sigma_{k}^{2}+\sigma_{k} \sum_{j \neq k} x_{j} \rho_{k, j} \sigma_{j}\right)=0
$$

We obtain two solutions. The first one is $x_{k}^{\prime}=0$ whereas the second one verifies:

$$
x_{k}^{\prime \prime}=-\frac{\sum_{j \neq k} x_{j} \rho_{k, j} \sigma_{j}}{\sigma_{k}}
$$

If $\rho_{k, j} \geq 0$ for all $j$, we have $\sum_{j \neq k} x_{j} \rho_{k, j} \sigma_{j} \geq 0$ because $x_{j} \geq 0$ and $\sigma_{j}>0$. It implies that $x_{k}^{\prime \prime} \leq 0$ meaning that $x_{k}^{\prime}=0$ is the unique positive solution. Finally the only way to have $x_{k}^{\prime \prime}>0$ is to have some negative correlations $\rho_{k, j}$. In this case, it implies that:

$$
\sum_{j \neq k} x_{j} \rho_{k, j} \sigma_{j}<0
$$

If we consider a universe of three assets, this constraint is verified for $k=3$ and a covariance matrix such that $\rho_{1,3}<0$ and $\rho_{2,3}<0$. For example, if $\sigma_{1}=20 \%, \sigma_{2}=10 \%, \sigma_{3}=5 \%$, $\rho_{1,2}=50 \%, \rho_{1,3}=-25 \%$ and $\rho_{2,3}=-25 \%$, the two solutions are $(33.33 \%, 66.67 \%, 0 \%)$ and $(20 \%, 20 \%, 40 \%)$ if the risk budgets are $(50 \%, 50 \%, 0 \%)$.

In practice, this second solution may not satisfy the investor. When he sets one risk budget to zero, he expects that he will not have the corresponding asset in his portfolio. That's why it is important to impose the strict constraint $b_{i}>0$. To summarize, the RB portfolio is the solution of the following mathematical problem:

$$
x^{\star}=\left\{x \in[0,1]^{n}: \sum_{i=1}^{n} x_{i}=1, x_{i} \cdot(\Sigma x)_{i}=b_{i} \cdot\left(x^{\top} \Sigma x\right)\right\}
$$

where $b \in] 0,1]^{n}$ and $\sum_{i=1}^{n} b_{i}=1$.

Remark 2 If we would like to impose that some risk budgets are equal to zero, we first have to reduce the universe of assets by excluding the assets corresponding to these zero risk contributions. Then, we solve the problem (3).

\subsection{Some properties of the RB portfolio}

In this section, we first analyse the RB portfolio in the two-asset case and the general case. Then, we show that the RB portfolio always exists and is unique. We also discuss the optimality of such a portfolio and illustrates what the solution becomes when some risk budgets are set to zero.

\footnotetext{
${ }^{7}$ We have of course $\rho_{i, i}=1$.
} 


\subsubsection{The two-asset case $(n=2)$}

We begin by analyzing the RB portfolio in the bivariate case. Let $\rho$ be the correlation, $x=(w, 1-w)$ be the vector of weights and $(b, 1-b)$ be the vector of risk budgets. The risk contributions are:

$$
\left(\begin{array}{l}
\mathrm{RC}_{1} \\
\mathrm{RC}_{2}
\end{array}\right)=\frac{1}{\sigma(x)}\left(\begin{array}{c}
w^{2} \sigma_{1}^{2}+w(1-w) \rho \sigma_{1} \sigma_{2} \\
(1-w)^{2} \sigma_{2}^{2}+w(1-w) \rho \sigma_{1} \sigma_{2}
\end{array}\right)
$$

The unique solution satisfying $0 \leq w \leq 1$ is:

$$
w^{\star}=\frac{(b-1 / 2) \rho \sigma_{1} \sigma_{2}-b \sigma_{2}^{2}+\sigma_{1} \sigma_{2} \sqrt{(b-1 / 2)^{2} \rho^{2}+b(1-b)}}{(1-b) \sigma_{1}^{2}-b \sigma_{2}^{2}+2(b-1 / 2) \rho \sigma_{1} \sigma_{2}}
$$

We notice that the solution depends on the correlation $\rho$ contrary to the ERC portfolio ${ }^{8}$ (Maillard et al., 2010). It is indeed a complex function of the volatilities $\sigma_{1}$ and $\sigma_{2}$, the correlation $\rho$ and the risk budget $b$.

In order to have some intuitions about the behavior of the solution, we consider some special cases. For example, if $\rho=0$, we obtain:

$$
w^{\star}=\frac{\sigma_{2} \sqrt{b}}{\sigma_{1} \sqrt{1-b}+\sigma_{2} \sqrt{b}}
$$

The weight of asset $i$ is then proportional to the square root of its risk budget and inversely proportional to its volatility. If $\rho=1$, the solution reduces to:

$$
w^{\star}=\frac{\sigma_{2} b}{\sigma_{1}(1-b)+\sigma_{2} b}
$$

In this case, the weight of asset $i$ is directly proportional to its risk budget. If $\rho=-1$, we notice also that the solution does not depend on the risk budgets:

$$
w^{\star}=\frac{\sigma_{2}}{\sigma_{1}+\sigma_{2}}
$$

because the volatility of the portfolio is equal to zero. We may also show that the weight of the asset $i$ is an increasing function of its risk budget and a decreasing function of its volatility. In Table 1 , we report some values taken by $w^{\star}$ with respect to the parameters $\rho$ and $b$, whereas the behavior of $w^{\star}$ is illustrated in Figure 1.

\subsubsection{The general case $(n>2)$}

In more general cases, where $n>2$, the number of parameters increases quickly, with $n$ individual volatilities and $n(n-1) / 2$ bivariate correlations. Because the two-asset case leads to a complex solution, finding an explicit solution for the general case is certainly impossible. But we can find some results that help us to understand the behavior of the RB portfolio.

Let us begin by assuming that the correlation matrix of assets is constant:

$$
\rho_{i, j}=\rho
$$

${ }^{8}$ This last one is obtained if $b=1 / 2$ :

$$
w^{\star}=\frac{\sigma_{2}}{\sigma_{1}+\sigma_{2}}
$$


Managing Risk Exposures using the Risk Budgeting Approach

Table 1: Weights $w^{\star}$ with respect to some values of $b$ and $\rho$

\begin{tabular}{|c|c|c|c|c|c|c|c|c|c|}
\hline \multirow{2}{*}{\multicolumn{2}{|c|}{$b$}} & \multicolumn{4}{|c|}{$\sigma_{2}=\sigma_{1}$} & \multicolumn{4}{|c|}{$\sigma_{2}=3 \times \sigma_{1}$} \\
\hline & & $20 \%$ & $50 \%$ & $70 \%$ & $90 \%$ & $20 \%$ & $50 \%$ & $70 \%$ & $90 \%$ \\
\hline \multirow{6}{*}{$\rho$} & $-50 \%$ & $41.9 \%$ & $50.0 \%$ & $55.2 \%$ & $61.6 \%$ & $68.4 \%$ & $75.0 \%$ & $78.7 \%$ & $82.8 \%$ \\
\hline & $0 \%$ & $33.3 \%$ & $50.0 \%$ & $60.4 \%$ & $75.0 \%$ & $60.0 \%$ & $75.0 \%$ & $82.1 \%$ & $90.0 \%$ \\
\hline & $25 \%$ & $29.3 \%$ & $50.0 \%$ & $63.0 \%$ & $80.6 \%$ & $55.5 \%$ & $75.0 \%$ & $83.6 \%$ & $92.6 \%$ \\
\hline & $50 \%$ & $25.7 \%$ & $50.0 \%$ & $65.5 \%$ & $84.9 \%$ & $51.0 \%$ & $75.0 \%$ & $85.1 \%$ & $94.4 \%$ \\
\hline & $75 \%$ & $22.6 \%$ & $50.0 \%$ & $67.8 \%$ & $87.9 \%$ & $46.7 \%$ & $75.0 \%$ & $86.3 \%$ & $95.6 \%$ \\
\hline & $90 \%$ & $21.0 \%$ & $50.0 \%$ & $69.1 \%$ & $89.2 \%$ & $44.4 \%$ & $75.0 \%$ & $87.1 \%$ & $96.1 \%$ \\
\hline
\end{tabular}

Figure 1: Evolution of the weight $w^{\star}$ (in \%) with respect to $b$ and $\rho$

$b=60 \%$
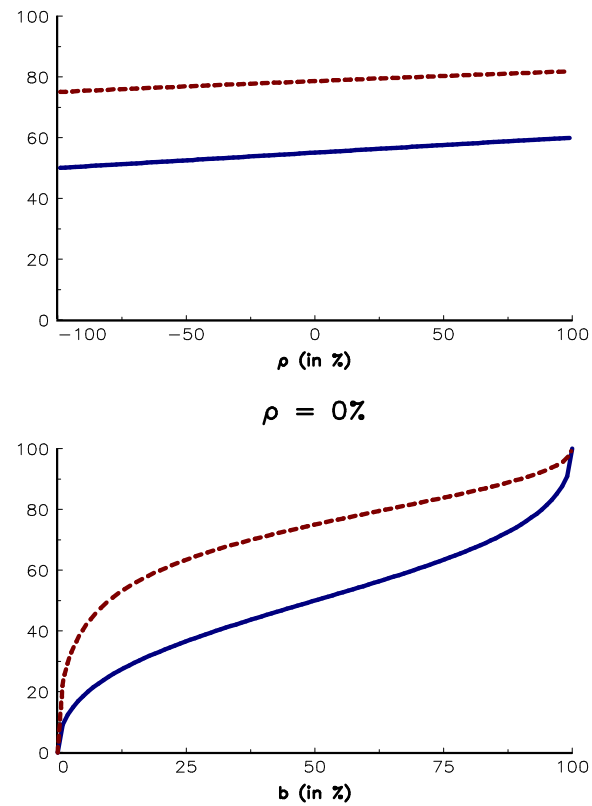

$\mathrm{b}=90 \%$
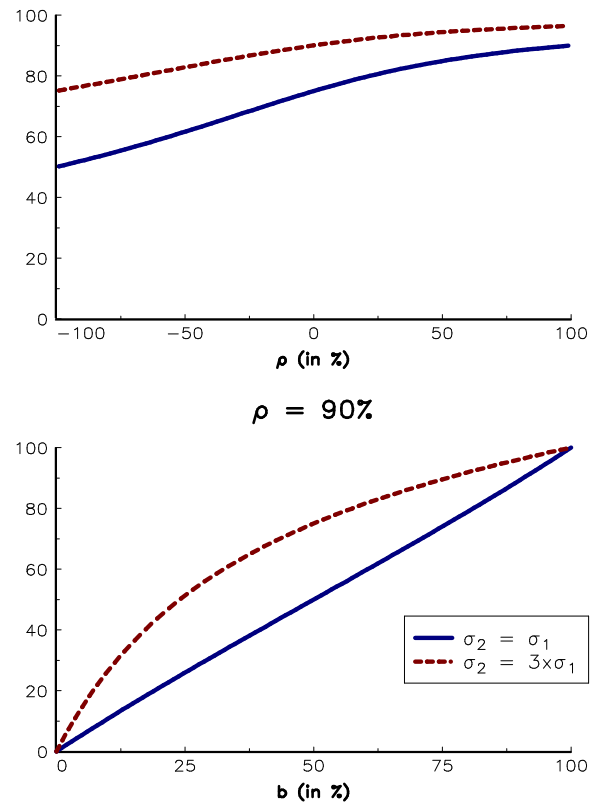

If we have no correlation, that is if $\rho=0$, the risk contribution of the asset $i$ becomes $\mathrm{RC}_{i}=$ $x_{i}^{2} \sigma_{i}^{2} / \sigma(x)$. The RB portfolio being defined by $\mathrm{RC}_{i}=b_{i} \sigma(x)$ for all $i$, some simple algebra shows that this is here equivalent to $\sqrt{b_{j}} x_{i} \sigma_{i}=\sqrt{b_{i}} x_{j} \sigma_{j}$. Coupled with the (normalizing) budget constraint $\sum_{i=1}^{n} x_{i}=1$, we deduce that:

$$
x_{i}=\frac{\sqrt{b_{i}} \sigma_{i}^{-1}}{\sum_{j=1}^{n} \sqrt{b_{j}} \sigma_{j}^{-1}}
$$

The weight allocated to the component $i$ is thus proportional to the square root of its risk budget and the inverse of its volatility. The higher (lower) the volatility of a component, the lower (higher) its weight in the RB portfolio. In the case of perfect correlation, that is $\rho=1$, the risk contribution of the asset $i$ becomes $\mathrm{RC}_{i}=x_{i} \sigma_{i}\left(\sum_{j=1}^{n} x_{j} \sigma_{j}\right) / \sigma(x)$. It comes 
that $b_{j} x_{i} \sigma_{i}=b_{i} x_{j} \sigma_{j}$. We then deduce that:

$$
x_{i}=\frac{b_{i} \sigma_{i}^{-1}}{\sum_{j=1}^{n} b_{j} \sigma_{j}^{-1}}
$$

The opposite of perfect correlation corresponds to the lower bound of the constant correlation matrix, which is reached for $\rho=-1 /(n-1)$. In this case, the volatility of the portfolio is equal to zero and the solution is the ERC portfolio ${ }^{9}$ :

$$
x_{i}=\frac{\sigma_{i}^{-1}}{\sum_{j=1}^{n} \sigma_{j}^{-1}}
$$

Unfortunately, there is no analytical solution in the general case when the constant correlation is different from 0,1 and $-1 /(n-1)$. Nevertheless, we could find an implicit form. We remind that the $\mathrm{RB}$ portfolio verifies the following equation in the case of the constant correlation matrix:

$$
x_{i} \sigma_{i}\left((1-\rho) x_{i} \sigma_{i}+\rho\left(\sum_{j=1}^{n} x_{j} \sigma_{j}\right)\right)=b_{i} \sigma^{2}(x)
$$

If we note $X_{i}=x_{i} \sigma_{i}$ and $B_{i}=b_{i} \sigma^{2}(x)$, the previous equation becomes $(1-\rho) X_{i}^{2}+$ $\rho X_{i}\left(\sum_{j=1}^{n} X_{j}\right)=B_{i}$. It implies that the general form of the solution is:

$$
x_{i}=\frac{f_{i}(\rho, b) \sigma_{i}^{-1}}{\sum_{j=1}^{n} f_{j}(\rho, b) \sigma_{j}^{-1}}
$$

The function $f_{i}(\rho, b)$ depends on the constant correlation $\rho$ and the vector $b$ of risk budgets. In particular, it verifies $f_{i}\left(-(n-1)^{-1}, b\right)=1, f_{i}(0, b)=\sqrt{b_{i}}, f_{i}(1, b)=b_{i}$ and $f_{i}\left(\rho, n^{-1} \mathbf{1}\right)=1$.

To illustrate the case of the constant correlation matrix, we have reported some simulations in Figure 2. We consider a universe of $n$ assets with same volatilities. We note $b_{1}$ the risk budget of the first asset. The risk budget of the other assets is uniform and equal to $\left(1-b_{1}\right) /(n-1)$. We notice the effect of the constant correlation $\rho$ on the weight $x_{1}$, in particular when the number $n$ of assets is large. When the number of assets is small (less than 10), the correlation $\rho$ has an impact only when it is small (less than 10\%). In other cases, the formula (5) is a good approximation of the solution ${ }^{10}$.

In other cases, it is not possible to find explicit solutions of the RB portfolio. But we can find a financial interpretation of the RB portfolio. We remind that the covariance between the returns of assets and the portfolio $x$ is equal to $\Sigma x$. The beta $\beta_{i}$ of the asset $i$ with respect to the portfolio $x$ is then defined as the ratio between the covariance term $(\Sigma x)_{i}$ and the variance $x^{\top} \Sigma x$ of the portfolio. $\beta_{i}$ indicates the sensitivity of the asset $i$ to the

\footnotetext{
${ }^{9}$ The proof is provided in Appendix A.1.

${ }^{10}$ Let $x_{i}(\rho)$ be the weight of the asset $i$ in the RB portfolio when the constant correlation is equal to $\rho$. A better approximation is given by the following rule:$$
x_{i}(\rho)=(1-\sqrt{\rho}) \times x_{i}(0)+\sqrt{\rho} \times x_{i}(1)
$$ 
Figure 2: Simulation of the weight $x_{1}$ when the correlation is constant

$n=5$
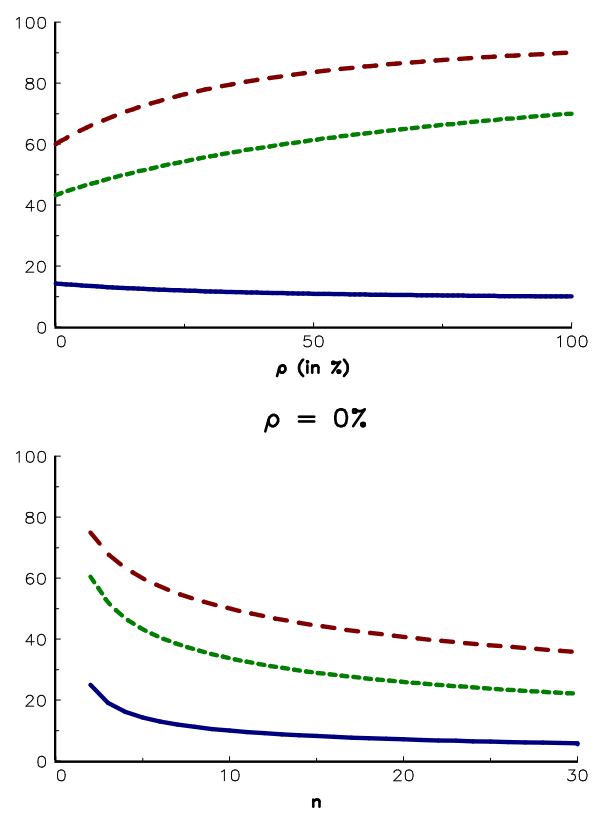

$n=30$
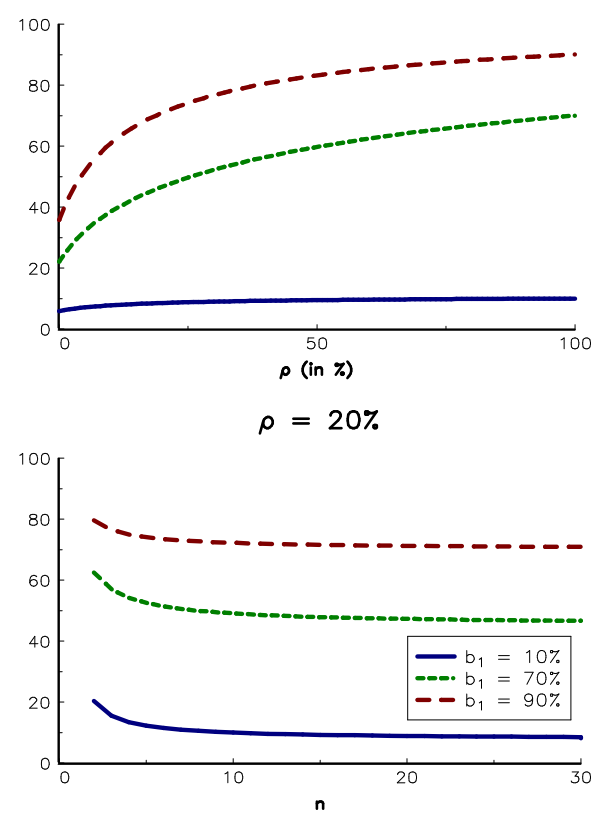

systematic (or market) risk, which is represented here by the portfolio $x$. It means that the risk contribution $\mathrm{RC}_{i}$ is equal to $x_{i} \beta_{i} \sigma(x)$. It follows that:

$$
b_{j} x_{i} \beta_{i}=b_{i} x_{j} \beta_{j}
$$

We finally deduce that:

$$
x_{i}=\frac{b_{i} \beta_{i}^{-1}}{\sum_{j=1}^{n} b_{j} \beta_{j}^{-1}}
$$

The weight allocated to the component $i$ is thus inversely proportional to its beta. However, contrary to the solutions (4), (5) and (6), this one is endogenous since $\beta_{i}$ is the beta of the asset $i$ with respect to the $\mathrm{RB}$ portfolio ${ }^{11}$.

\subsubsection{Existence and uniqueness of the RB portfolio}

As for the ERC portfolio, we may use the SQP (Sequential Quadratic Programming) algorithm to find the RB portfolio ${ }^{12}$ :

$$
x^{\star}=\arg \min \sum_{i=1}^{n}\left(\frac{x_{i}\left(\sum x\right)_{i}}{\sum_{j=1}^{n} x_{j}(\Sigma x)_{j}}-b_{i}\right)^{2}
$$

$$
\text { u.c. } \mathbf{1}^{\top} x=1 \text { and } \mathbf{0} \leq x \leq \mathbf{1}
$$

\footnotetext{
${ }^{11}$ With the equation (7), we have a new interpretation of the function $f_{i}(\rho, b)$ :

$$
f_{i}(\rho, b)=\frac{b_{i}}{\beta_{i}} \sigma_{i}
$$

It is the product of the risk budget and the volatility scaled by the beta.

${ }^{12}$ From a numerical point of view, it is preferable to solve the system without the constraint $\mathbf{1}^{\top} x=1$ and then to rescale the solution.
} 
An alternative to the previous algorithm is to consider the following optimization problem:

$$
\begin{aligned}
y^{\star}= & \arg \min \sqrt{y^{\top} \Sigma y} \\
\text { u.c. } & \left\{\begin{array}{l}
\sum_{i=1}^{n} b_{i} \ln y_{i} \geq c \\
y \geq \mathbf{0}
\end{array}\right.
\end{aligned}
$$

with $c$ an arbitrary constant. In this case, the program is similar to a variance minimization problem subject to a constraint. The constant $c$ could be adjusted to obtain $\sum_{i=1}^{n} y_{i}^{*}=1$ implying that the RB portfolio is expressed as $x_{i}^{\star}=y_{i}^{*} / \sum_{i=1}^{n} y_{i}^{*}$ (see Appendix A.2).

The formulation (9) is very interesting because it demonstrates that the RB portfolio specified by the mathematical system (3) exists and is unique as long as the covariance matrix $\Sigma$ is positive-definite. Indeed, it corresponds to the minimization program of a quadratic function (a convex function) with a convex constraint ${ }^{13}$.

\subsubsection{Comparing the risk budgeting portfolio with the weight budgeting port- folio}

Maillard et al. (2010) shows that the ERC portfolio is located between the minimum variance portfolio and the equally-weighted portfolio. We could extend this result to the risk budgeting portfolio. Let $x_{\mathrm{wb}}$ be the weight budgeting (WB) porfolio, that is $x_{i}=b_{i}$. We have:

$$
\begin{array}{ll}
x_{i} / b_{i}=x_{j} / b_{j} & (\mathrm{wb}) \\
\partial_{x_{i}} \sigma(x)=\partial_{x_{j}} \sigma(x) & (\mathrm{mv}) \\
x_{i} \partial_{x_{i}} \sigma(x) / b_{i}=x_{j} \partial_{x_{j}} \sigma(x) / b_{j} & (\mathrm{rb})
\end{array}
$$

Thus, a RB portfolio may be viewed as a portfolio located between the MV and WB portfolios. To elaborate further this point of view, let us consider a modified version of the optimization problem (9):

$$
\begin{aligned}
x^{\star}(c)= & \arg \min \sqrt{x^{\top} \Sigma x} \\
\text { u.c. } & \left\{\begin{array}{l}
\sum_{i=1}^{n} b_{i} \ln x_{i} \geq c \\
\mathbf{1}^{\top} x=1 \\
x \geq \mathbf{0}
\end{array}\right.
\end{aligned}
$$

In order to get the RB portfolio, one minimizes the volatility of the portfolio subject to an additional constraint, $\sum_{i=1}^{n} b_{i} \ln x_{i} \geq c$ where $c$ is a constant being determined by the RB portfolio. In Appendix A.3, we prove that the volatility $\sigma\left(x^{\star}(c)\right)$ is an increasing function of $c$. Two polar cases can be defined with $c=-\infty$ for which one gets the MV portfolio and $c=\sum_{i=1}^{n} b_{i} \ln b_{i}$ where one gets the WB portfolio. We finally deduce that the volatility of the risk budgeting portfolio is between the volatility of the minimum variance portfolio and the volatility of the weight budgeting portfolio:

$$
\sigma_{\mathrm{mv}} \leq \sigma_{\mathrm{rb}} \leq \sigma_{\mathrm{wb}}
$$

\subsubsection{Optimality}

Like the ERC portfolio, the risk budgeting approach is an heuristic asset allocation method. In finance, we like to derive optimal portfolios from the maximisation problem of an utility function. For example, Maillard et al. (2010) show that the ERC portfolio corresponds to

\footnotetext{
${ }^{13}$ Because $b_{i}>0$.
} 
the tangency portfolio when the correlation is the same and when the assets have the same Sharpe ratio. For the RB portfolio, it is more difficult to find such properties ${ }^{14}$.

Let us consider the quadratic utility function $x^{\top} \mu-\phi x^{\top} \Sigma x$ corresponding to the Markowitz criteria. The portfolio $x$ is optimal if the vector of expected returns satisfies this relation$\operatorname{ship}^{15}$ :

$$
\tilde{\mu}=\frac{2}{\phi} \Sigma x
$$

If the $\mathrm{RB}$ portfolio is optimal, the performance contribution $\mathrm{PC}_{i}$ of the asset $i$ is then proportional to its risk budgets:

$$
\begin{aligned}
\mathrm{PC}_{i} & =x_{i} \tilde{\mu}_{i} \\
& =\frac{2}{\phi} x_{i}(\Sigma x)_{i} \\
& \propto b_{i}
\end{aligned}
$$

The specification of the risk budgets allows us to decide not only which amount of risk to invest in one asset, but also which amount of expected performance to attribute to this asset.

We consider a universe of 4 risky assets. Volatilities are respectively 10\%, 20\%, $30 \%$ and $40 \%$ and the correlation matrix is equal to:

$$
\rho=\left(\begin{array}{cccc}
1.00 & & & \\
0.80 & 1.00 & & \\
0.20 & 0.20 & 1.00 & \\
0.20 & 0.20 & 0.50 & 1.00
\end{array}\right)
$$

We suppose that the risk budgets are inversely proportional to the volatilities and $\phi=0.4$. We verify that performance contributions are proportional to risk budgets:

\begin{tabular}{r|rrrrr}
$i$ & \multicolumn{1}{|c}{$x_{i}$} & $\partial_{x_{i}} \sigma(x)$ & \multicolumn{1}{c}{$\mathrm{RC}_{i}$} & \multicolumn{1}{c}{$\tilde{\mu}_{i}$} & $\mathrm{PC}_{i}$ \\
\hline 1 & $64.9 \%$ & $8.8 \%$ & $48.0 \%$ & $5.2 \%$ & $48.0 \%$ \\
2 & $17.2 \%$ & $16.6 \%$ & $24.0 \%$ & $9.8 \%$ & $24.0 \%$ \\
3 & $11.2 \%$ & $16.9 \%$ & $16.0 \%$ & $10.0 \%$ & $16.0 \%$ \\
4 & $6.7 \%$ & $21.4 \%$ & $12.0 \%$ & $12.7 \%$ & $12.0 \%$
\end{tabular}

\subsubsection{Back to the case when some risk budgets are set to zero}

The analysis of the optimization problem (10) permits also to clarify the disturbing point discussed in Section 2.2 about the possibility of several solutions when some assets have a null risk budget. By construction, the formulation (10) implies necessarily that the solution exists and is unique even if $b_{i}=0$ for some assets. In Appendix A.2, we have specify this solution. Let $\mathcal{N}$ be the set of assets such that $b_{i}=0$. The solution $\mathcal{S}_{1}$ of the optimization problem (10) satisfies the following relationships:

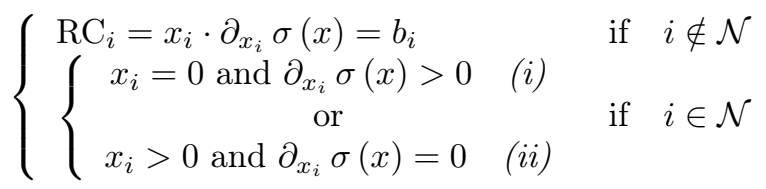

\footnotetext{
${ }^{14}$ The RB portfolio could be written as the solution of a maximisation problem with an objective function equal to $\ln \left(\prod_{i=1}^{n} x_{i}^{b_{i}}\right)$. Interpreting this problem as a utility maximization problem is attractive, but the objective function does not present right properties to be a utility function.

${ }^{15}$ We use the notation $\tilde{\mu}$ to specify that it is the market price of expected returns with respect to the current portfolio.
} 
Managing Risk Exposures using the Risk Budgeting Approach

The conditions $(i)$ and $(i i)$ are mutually exclusive for one asset $i \in \mathcal{N}$, but not necessarily for all the assets $i \in \mathcal{N}$.

If we consider the system (2) such that $b_{i}=0$ for $i \in \mathcal{N}$, we can clarify the number of solutions by just analysing the solution of the optimization problem (10). Let $\mathcal{N}=\mathcal{N}_{1} \sqcup \mathcal{N}_{2}$ where $\mathcal{N}_{1}$ is the set of assets verifying the condition $(i)$ and $\mathcal{N}_{2}$ is the set of assets verifying the condition $(i i)$. The number of solutions ${ }^{16}$ is equal to $2^{m}$ where $m=\left|\mathcal{N}_{1}\right|$ is the cardinality of $\mathcal{N}_{1}$. Indeed, it is the number of $k$-combinations for all $k=0,1, \ldots, m$.

Suppose that $m=0$. In this case, the solution $\mathcal{S}_{1}$ verifies $x_{i}=0$ if $b_{i}=0$. It corresponds to the solution expected by the investor. If $m \geq 1$, there are several solutions, in particular the solution $\mathcal{S}_{1}$ given by the optimization problem (10) and another solution $\mathcal{S}_{2}$ with $x_{i}=0$ for all assets such that $b_{i}=0$. We then obtain a paradox because even if $\mathcal{S}_{2}$ is the solution expected by the investor, the only acceptable solution is $\mathcal{S}_{1}$. The argument is very simple. Suppose that you impose $b_{i}=\varepsilon_{i}$ with $\varepsilon_{i}>0$ a small number for $i \in \mathcal{N}$. In this case, you obtain a unique solution. If $\varepsilon_{i} \rightarrow 0$, this solution will converge to $\mathcal{S}_{1}$, not to $\mathcal{S}_{2}$ or all the others solutions $\mathcal{S}_{j}$ for $j=3, \ldots, 2^{m}$.

Table 2: Solution when the risk budget $b_{3}$ is equal to 0

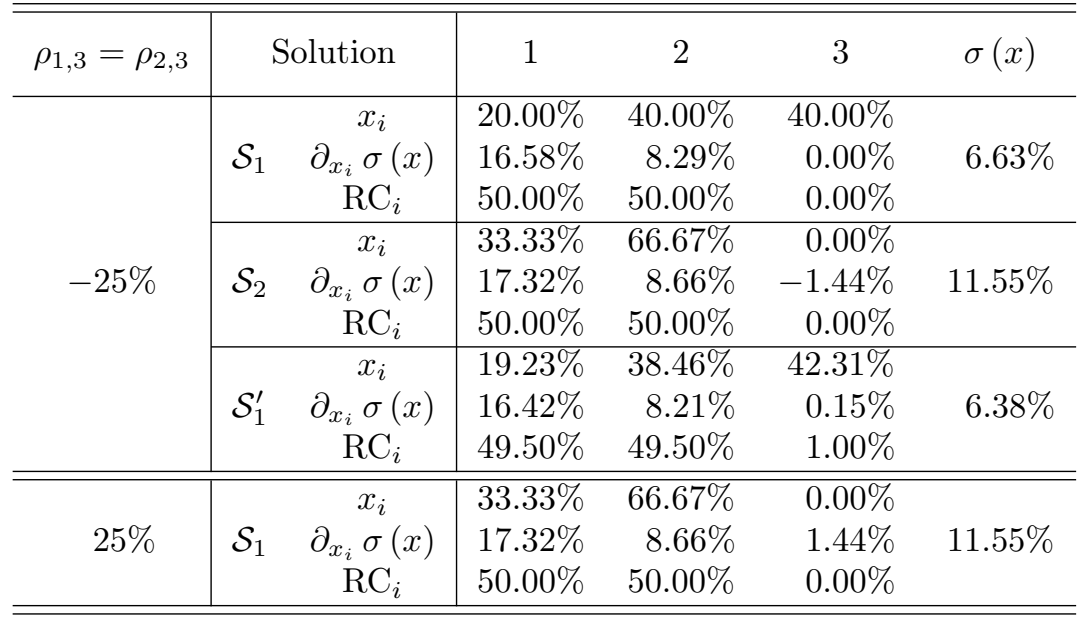

Let us illustrate this paradox with the example given in Section 2.2. Results are reported in Table 2 when the risk budgets are $b_{1}=50 \%, b_{2}=50 \%$ and $b_{3}=0 \%$. If $\rho_{1,3}=\rho_{2,3}=$ $-25 \%$, we obtain two solutions $\mathcal{S}_{1}$ and $\mathcal{S}_{2}$. For the first solution, we have $x_{1}=20 \%$, $x_{2}=40 \%$ and $x_{3}=40 \%$, whereas the weights for the second solution are respectively $x_{1}=33.33 \%, x_{2}=66.67 \%$ and $x_{3}=0 \%$. We verify that the marginal risk $\partial_{x_{3}} \sigma(x)$ is negative for the second solution indicating that it could not be a solution of the optimization problem. That's why the volatility of this second solution (11.55\%) is larger than the volatility to the first solution $(6.63 \%)$. We also notice that if we perturb slightly the risk budgets $\left(b_{1}=49.5 \%, b_{2}=49.5 \%\right.$ and $\left.b_{3}=1 \%\right)$, the solution $\mathcal{S}_{1}^{\prime}$ is closer to $\mathcal{S}_{1}$ than to $\mathcal{S}_{2}$. It confirms that the first solution $\mathcal{S}_{1}$ is more acceptable. If all the correlations are positive $\left(\rho_{1,3}=\rho_{2,3}=25 \%\right)$, we do not face this problem. The unique solution is $x_{1}=33.33 \%$, $x_{2}=66.67 \%$ and $x_{3}=0 \%$ and we verify that the marginal risk $\partial_{x_{3}} \sigma(x)$ is positive. In

\footnotetext{
${ }^{16}$ To be more correct, $2^{m}$ is the maximum number of solutions. But the case when fixing some weights of $\mathcal{N}_{1}$ to zero implies that other assets of $\mathcal{N}_{1}$ have a weight equal to zero has a small probability to occur.
} 
Figure 3, we have represented the evolution of the volatility of the portfolio $\left(50 \%, 50 \%, x_{3}\right)$ with respect to the weight $x_{3}$. If $\rho_{1,3}=\rho_{2,3}=-25 \%$, the volatility is decreasing around $x_{3}=0$ meaning that the minimum is after $x_{3}=0$. If $\rho_{1,3}=\rho_{2,3}=25 \%$, the volatility is increasing around $x_{3}=0$ meaning that the minimum is before $x_{3}=0$. This figure illustrates that the solution verifies $x_{3}>0$ in the first case and $x_{3}=0$ in the second case.

Figure 3: Evolution of the volatility with respect to $x_{3}$

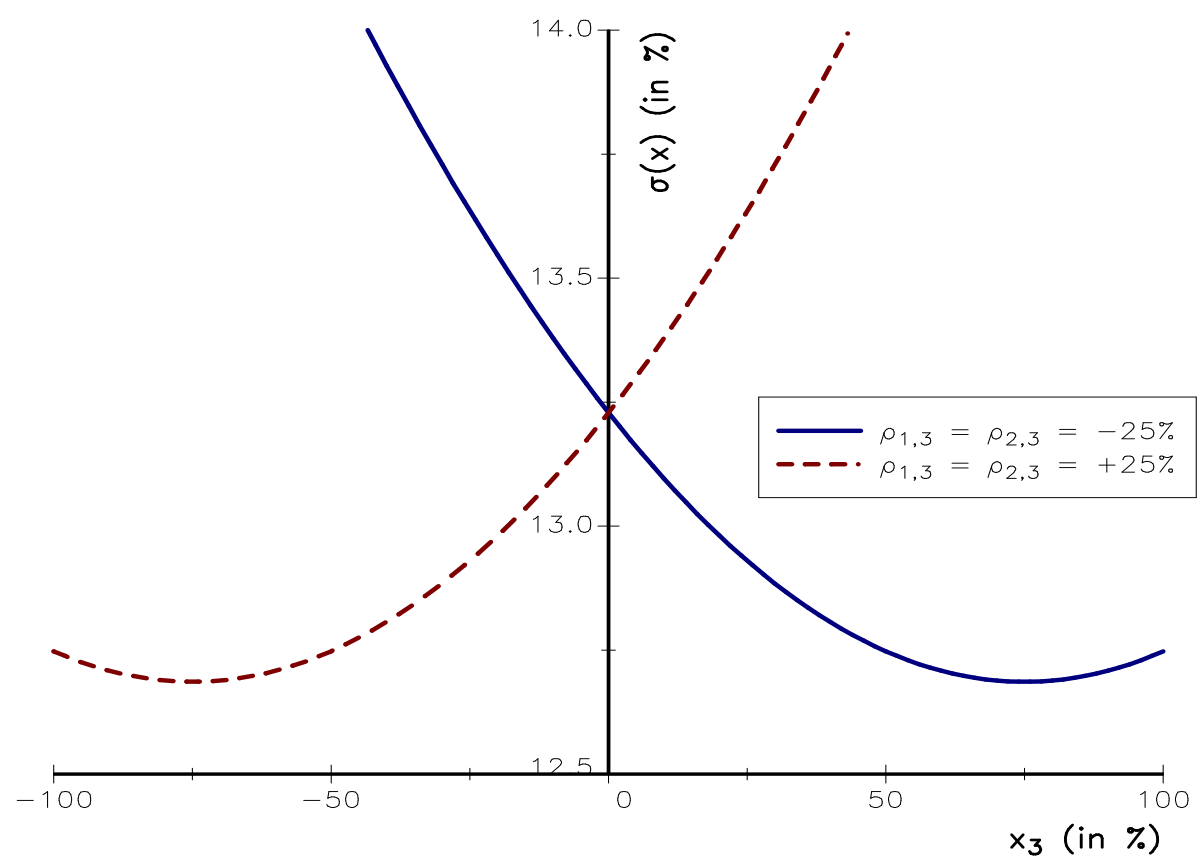

Let us add a fourth asset to the previous universe with $\sigma_{4}=10 \%, \rho_{1,4}=\rho_{2,4}=-25 \%$ and $\rho_{3,4}=50 \%$. Table 3 gives the results when the risk budgets are $b_{1}=50 \%, b_{2}=50 \%$, $b_{3}=0 \%$ and $b_{4}=0 \%$. If we analyze the solution $\mathcal{S}_{1}$, we notice that the number $m$ of assets such that both the marginal risk and the risk budgets are zero is equal to 2 . That's why we obtain $2^{2}=4$ solutions. Another way to determine the number of solutions is to compute the solution $\mathcal{S}_{2}$ desired by the investor and set $m$ equal to the number of assets such that the marginal risk is strictly negative when the risk budget is equal to zero.

\subsection{Generalization to other risk measures}

We could show that some previous results are valid to convex risk measures $\mathcal{R}$ that satisfy the Euler decomposition, because it suffices to replace the marginal volatility by the marginal risk in the mathematical proofs. The optimization problem (9) becomes:

$$
\begin{aligned}
y^{\star}= & \arg \min \mathcal{R}(y) \\
\text { u.c. } & \left\{\begin{array}{l}
\sum_{i=1}^{n} b_{i} \ln y_{i} \geq c \\
y \geq \mathbf{0}
\end{array}\right.
\end{aligned}
$$

It is then easy to show that if the risk budgets $b_{i}$ are strictly positive, the RB portfolio exists and is unique whereas there may be several solutions if some risk budgets are null. Using 
Managing Risk Exposures using the Risk Budgeting Approach

Table 3: Solution when the risk budgets $b_{3}$ and $b_{4}$ are equal to 0

\begin{tabular}{cc|rrrrr}
\hline \hline \multicolumn{2}{c|}{ Solution } & \multirow{2}{*}{1} & \multicolumn{1}{c}{2} & \multicolumn{1}{c}{3} & \multicolumn{1}{c}{4} & $\sigma(x)$ \\
\hline & $x_{i}$ & $20.00 \%$ & $40.00 \%$ & $26.67 \%$ & $13.33 \%$ & \\
$\mathcal{S}_{1}$ & $\partial_{x_{i}} \sigma(x)$ & $16.33 \%$ & $8.16 \%$ & $0.00 \%$ & $0.00 \%$ & $6.53 \%$ \\
& $\mathrm{RC}_{i}$ & $50.00 \%$ & $50.00 \%$ & $0.00 \%$ & $0.00 \%$ & \\
\hline & $x_{i}$ & $33.33 \%$ & $66.67 \%$ & $0.00 \%$ & $0.00 \%$ & \\
$\mathcal{S}_{2}$ & $\partial_{x_{i}} \sigma(x)$ & $17.32 \%$ & $8.66 \%$ & $-1.44 \%$ & $-2.89 \%$ & $11.55 \%$ \\
& $\mathrm{RC}_{i}$ & $50.00 \%$ & $50.00 \%$ & $0.00 \%$ & $0.00 \%$ & \\
\hline & $x_{i}$ & $20.00 \%$ & $40.00 \%$ & $40.00 \%$ & $0.00 \%$ & \\
$\mathcal{S}_{3}$ & $\partial_{x_{i}} \sigma(x)$ & $16.58 \%$ & $8.29 \%$ & $0.00 \%$ & $-1.51 \%$ & $6.63 \%$ \\
& $\mathrm{RC}_{i}$ & $50.00 \%$ & $50.00 \%$ & $0.00 \%$ & $0.00 \%$ & \\
\hline & $x_{i}$ & $25.00 \%$ & $50.00 \%$ & $0.00 \%$ & $25.00 \%$ & \\
$\mathcal{S}_{4}$ & $\partial_{x_{i}} \sigma(x)$ & $16.58 \%$ & $8.29 \%$ & $-0.75 \%$ & $0.00 \%$ & $8.29 \%$ \\
& $\mathrm{RC}_{i}$ & $50.00 \%$ & $50.00 \%$ & $0.00 \%$ & $0.00 \%$ & \\
\hline \hline
\end{tabular}

the same proof by replacing the marginal volatility by the marginal risk, we could also show that the risk of the RB portfolio is between the risk of the minimum risk portfolio and the risk of the weight budgeting portfolio:

$$
\mathcal{R}\left(x_{\min }\right) \leq \mathcal{R}\left(x_{\mathrm{rb}}\right) \leq \mathcal{R}\left(x_{\mathrm{wb}}\right)
$$

And if the RB portfolio is optimal in the sense of the following utility function $U(x)=$ $x^{\top} \mu-\phi \mathcal{R}^{2}(x)$, the performance contribution is equal to the risk contribution.

\section{Some illustrations}

The risk budgeting approach is an allocation method extensively used in asset management. There is also a huge literature on this subject (see for example the books of Rahl (2000), Grinold and Kahn (2000), Meucci (2005) or Scherer (2007)). For a very long time, the risk budgeting technique has been applied to a universe of multi-assets classes to manage and monitor the portfolio risk of large and sophisticated institutional investors like pension funds (Sharpe, 2002). More recently, this technique has been used to build alternative indexes in order to provide new benchmarks than the traditional market-cap indexes.

\subsection{Comparing risk budgeting portfolios with mean-variance or other heuristic portfolios}

In this section, we first compare RB portfolios with mean-variance optimized (MVO) portfolios. Then, we give new interpretation of some heuristic portfolios as RB portfolios.

\subsubsection{What are the main differences between RB and MVO portfolios?}

The main difference between RB and MVO portfolios is that the last ones are based on optimization techniques. It implies that MVO portfolios are very sensitive to the inputs:

"The indifference of many investment practitioners to mean-variance optimization technology, despite its theoretical appeal, is understandable in many cases. 
Managing Risk Exposures using the Risk Budgeting Approach

The major problem with $M V$ optimization is its tendency to maximize the effects of errors in the input assumptions. Unconstrained MV optimization can yield results that are inferior to those of simple equal-weighting schemes" (Michaud, 1989).

For MVO portfolios, the risk approach is marginal and the quantity of interest to study is the marginal volatility. For RB portfolios, the risk approach becomes global by mixing the marginal volatility and the weight. Let us illustrate these two ways to consider risk by an example. We consider three assets with $\mu_{1}=8 \%, \mu_{2}=8 \%, \mu_{3}=5 \%, \sigma_{1}=20 \%, \sigma_{2}=21 \%$, $\sigma_{3}=10 \%$. We assume that the correlation between the asset returns is uniform and is equal to $80 \%$. The MVO portfolio is equal to $x_{1}=38.3 \%, x_{2}=20.2 \%$ and $x_{3}=41.5 \%$ if we target a portfolio volatility of $15 \%$. In this case, the risk budgets are respectively $b_{1}=49.0 \%$, $b_{2}=25.8 \%$ and $b_{3}=25.2 \%$. Of course, the RB portfolio corresponding to these risk budgets is exactly the MVO portfolio. In Table 4, we have reported how these two portfolios evolve when we change slightly the inputs ${ }^{17}$. For example, if the uniform correlation is $90 \%$, the MVO and RB portfolios become $(44.6 \%, 8.9 \%, 46.5 \%)$ and $(38.9 \%, 20.0 \%, 41.1 \%)$. The MVO portfolio is thus very sensitive to the input parameters whereas the RB portfolio is more robust. In a dynamic investment strategy, the input parameters will change from one period to another period. Because $\left(\mu_{t+1}, \Sigma_{t+1}\right)$ will certainly be different from $\left(\mu_{t}, \Sigma_{t}\right)$, it would imply that a dynamic strategy based on MVO portfolios will generate a higher turnover than a dynamic strategy based on RB portfolios. This lack of robustness penalizes so much MVO portfolios that they are not used in practice without introducing some constraints.

Table 4: Sensitivity of MVO and RB portfolios to input parameters

\begin{tabular}{rr|rrrrrr}
\hline \hline \multicolumn{2}{c|}{\begin{tabular}{r|rrrrrr}
$c$ \\
$\sigma_{2}$
\end{tabular}} & & $70 \%$ & $90 \%$ & & $90 \%$ & \\
$\mu_{1}$ & & & & & $18 \%$ & $18 \%$ & \\
\hline \multirow{2}{*}{ MVO } & $x_{1}$ & $38.3 \%$ & $38.3 \%$ & $44.6 \%$ & $13.7 \%$ & $0.0 \%$ & $56.4 \%$ \\
& $x_{2}$ & $20.2 \%$ & $25.9 \%$ & $8.9 \%$ & $56.1 \%$ & $65.8 \%$ & $0.0 \%$ \\
& $x_{3}$ & $41.5 \%$ & $35.8 \%$ & $46.5 \%$ & $30.2 \%$ & $34.2 \%$ & $43.6 \%$ \\
\hline \multirow{3}{*}{$\mathrm{RB}$} & $x_{1}$ & $38.3 \%$ & $37.7 \%$ & $38.9 \%$ & $37.1 \%$ & $37.7 \%$ & $38.3 \%$ \\
& $x_{2}$ & $20.2 \%$ & $20.4 \%$ & $20.0 \%$ & $22.8 \%$ & $22.6 \%$ & $20.2 \%$ \\
& $x_{3}$ & $41.5 \%$ & $41.9 \%$ & $41.1 \%$ & $40.1 \%$ & $39.7 \%$ & $41.5 \%$ \\
\hline \hline
\end{tabular}

The problem of MVO portfolios comes from the solution structure, because optimal solutions are of the following form:

$$
x^{\star} \propto \Sigma^{-1} \mu
$$

The important quantity is then $\mathcal{I}=\Sigma^{-1}$, which is called the information matrix. We know that the eigendecomposition of $\mathcal{I}$ is related to the eigendecomposition of $\Sigma$ in the following way: the eigenvectors are the same and the eigenvalues of $\mathcal{I}$ are equal to the inverse of the eigenvalues of $\Sigma$ :

$$
\begin{aligned}
V_{i}(\mathcal{I}) & =V_{n-i}(\Sigma) \\
\lambda_{i}(\mathcal{I}) & =\frac{1}{\lambda_{n-i}(\Sigma)}
\end{aligned}
$$

For example, if we consider our previous example, we obtain the results in Table 5. It implies

\footnotetext{
${ }^{17}$ For the MVO portfolio, the objective is to maximize the expected return for a given volatility of $15 \%$, whereas the objective of the RB portfolio is to match the risk budgets $(49.0 \%, 25.8 \%, 25.2 \%)$.
} 
Managing Risk Exposures using the Risk Budgeting Approach

Table 5: Eigendecomposition of the covariance and information matrices

\begin{tabular}{|c|c|c|c|c|c|c|}
\hline \multirow[b]{2}{*}{ Asset / Factor } & \multicolumn{3}{|c|}{ Covariance matrix $\Sigma$} & \multicolumn{3}{|c|}{ Information matrix $\mathcal{I}$} \\
\hline & 1 & 2 & 3 & 1 & 2 & 3 \\
\hline 1 & $65.35 \%$ & $-72.29 \%$ & $-22.43 \%$ & $-22.43 \%$ & $-72.29 \%$ & $65.35 \%$ \\
\hline 2 & $69.38 \%$ & $69.06 \%$ & $-20.43 \%$ & $-20.43 \%$ & $69.06 \%$ & $69.38 \%$ \\
\hline 3 & $30.26 \%$ & $-2.21 \%$ & $95.29 \%$ & $95.29 \%$ & $-2.21 \%$ & $30.26 \%$ \\
\hline Eigenvalue & $8.31 \%$ & $0.84 \%$ & $0.26 \%$ & 379.97 & 119.18 & 12.04 \\
\hline$\%$ cumulated & $88.29 \%$ & $97.20 \%$ & $100.00 \%$ & $74.33 \%$ & $97.65 \%$ & $100.00 \%$ \\
\hline
\end{tabular}

that MVO portfolios $x^{\star}$ depends also on the most important factors of $\mathcal{I}$, that is the less important factors of $\Sigma$. But the last eigenfactors of a covariance matrix represent generally noise or very specific factors. It explains why MVO portfolios are not robust to input parameters, because a small change in the covariance matrix will change dramatically the last factor. To solve this type of problem, market practitioners introduce some regularization techniques:

- regularization of the objective function by using resampling techniques (Tütüncü and Koenig, 2004);

- regularization of the covariance matrix:

- Factor analysis based on PCA, ICA, etc. (Hyvärinen and Oja, 2000);

- Shrinkage methods (Ledoit and Wolf, 2003);

- Random matrix theory (Laloux et al., 1999);

- etc.

- regularization of the program specification by introducing some constraints on weights.

Even if the first two techniques are used, practitioners generally add constraints on weights in order to obtain a more satisfactory solution from a financial point of view. Jagannathan and Ma (2003) have shown that it is equivalent to shrink the covariance matrix. Introducing some constraints on weights may also lead to a covariance matrix that could be very different from the original one (Roncalli, 2011). With RB portfolios, we don't need to add constraints to obtain a satisfactory solution. It is certainly the main difference between MVO and RB portfolios.

\subsubsection{New interpretation of the EW, MV, MDP and ERC portfolios}

The risk budgeting approach leads us to a new interpretation of some specific portfolios:

- The equally-weighted (EW) portfolio could be viewed as a risk budgeting portfolio when the risk budget is proportional to the beta of the asset. Using the result (7), $b_{i}=\beta_{i} / n$ implies that:

$$
x_{i}=\frac{b_{i} \beta_{i}^{-1}}{\sum_{j=1}^{n} b_{j} \beta_{j}^{-1}}=\frac{1}{n}
$$

- The minimum variance portfolio is a risk budgeting portfolio when the risk budget is equal to the weight of the asset:

$$
b_{i}=x_{i}
$$


Let us consider an iterated portfolio $\left(x_{1}^{(t)}, \ldots, x_{1}^{(t)}\right)$ where $t$ represents the iteration. The portfolio is defined such that the risk budget $b_{i}^{(t)}$ of the asset $i$ at iteration $t$ corresponds to the weight $x_{i}^{(t-1)}$ at iteration $t-1$. If the portfolio $\left(x_{1}^{(t)}, \ldots, x_{1}^{(t)}\right)$ admits a limit when $t \rightarrow \infty$, it is equal to the minimum variance portfolio.

- The MDP portfolio of Choueifaty and Coignard (2008) et al. (2010) is the RB portfolio such that the risk budgets are proportional to the product of the weight of the asset and its volatility ${ }^{18}$ :

$$
b_{i} \propto x_{i} \sigma_{i}
$$

- Maillard et al. (2010) defines the ERC portfolio as the portfolio such that the risk contribution of all assets is the same. Using the previous results, it is also the optimal portfolio such that the performance contribution of all assets is the same.

Let us consider an example with 5 assets. The volatilities are respectively $\sigma_{1}=10 \%$, $\sigma_{2}=20 \%, \sigma_{3}=30 \%, \sigma_{4}=40 \%$, and $\sigma_{5}=30 \%$ whereas the correlation matrix is equal to:

$$
\rho=\left(\begin{array}{rrrrr}
1.00 & & & & \\
0.80 & 1.00 & & & \\
0.00 & 0.00 & 1.00 & & \\
0.00 & 0.00 & -0.50 & 1.00 & \\
0.00 & 0.00 & -0.20 & 0.80 & 1.00
\end{array}\right)
$$

Results are reported in Table 6 . The second column $x_{i}$ indicates the weight of asset $i$ in the portfolio, $\partial_{x_{i}} \sigma(x)$ corresponds to its marginal volatility, $\mathrm{RC}_{i}$ is its risk contribution and $\beta_{i}$ is its beta. $\mathrm{VC}_{i}=x_{i} \sigma_{i}$ corresponds to the volatility contribution. It could be interpreted as the risk contribution of the asset in the case of a perfect correlation $\left(\rho_{i, j}=1\right)$ between asset returns:

$$
\begin{aligned}
\mathrm{VC}_{i} & =\frac{x_{i}\left(\sum x\right)_{i}}{\sqrt{x^{\top} \sum x}} \\
& =\frac{x_{i} \sigma_{i}\left(\sum_{j=1}^{n} x_{j} \sigma_{j}\right)}{\sqrt{\left(\sum_{j=1}^{n} x_{j} \sigma_{j}\right)^{2}}} \\
& =x_{i} \sigma_{i}
\end{aligned}
$$

The seventh column gives the market price (or implied expected return) $\tilde{\mu}_{i}$ of the asset ${ }^{19}$ whereas the last column indicates the performance contribution $\mathrm{PC}_{i}=x_{i} \tilde{\mu}_{i} /\left(\sum_{j=1}^{n} x_{j} \tilde{\mu}_{j}\right)$ if the portfolio is optimal.

We verify that the risk contributions of the EW portfolio are proportional to the beta, that those of the $\mathrm{MV}^{20}$ and MDP portfolios are equal respectively to the weights and the volatility contributions and that the performance contributions of the ERC portfolio are equal. This simple example shows how different could be these heuristic portfolios in terms of weights but also in terms of risk metrics (risk contribution, beta, etc.).

\footnotetext{
${ }^{18} \mathrm{As}$ for the MV portfolio, this solution is endogenous because it depends on the weights of the portfolio. ${ }^{19}$ It has been calibrated in order that the average market price of the five assets is equal to $8 \%$.

${ }^{20}$ If we consider the iterated procedure presented above to find the MV portfolio with the EW portfolio as the starting portfolio, the convergence is achieved after 13 iterations for a tolerance of $1 \mathrm{bp}$ in terms of volatility or mean square error.
} 
Managing Risk Exposures using the Risk Budgeting Approach

Table 6: EW, MV, MDP and ERC portfolios

\begin{tabular}{r|rrrrrrrr}
\hline \hline & $i$ & \multicolumn{1}{c}{$x_{i}$} & $\partial_{x_{i}} \sigma(x)$ & \multicolumn{1}{c}{$\mathrm{RC}_{i}$} & $\beta_{i}$ & \multicolumn{1}{c}{$\mathrm{VC}_{i}$} & \multicolumn{1}{c}{$\tilde{\mu}_{i}$} & $\mathrm{PC}_{i}$ \\
\hline \multirow{5}{*}{ EW } & 1 & $20.00 \%$ & $3.84 \%$ & $5.68 \%$ & 0.28 & $7.69 \%$ & $2.27 \%$ & $5.68 \%$ \\
& 2 & $20.00 \%$ & $8.27 \%$ & $12.23 \%$ & 0.61 & $15.38 \%$ & $4.89 \%$ & $12.23 \%$ \\
& 3 & $20.00 \%$ & $1.77 \%$ & $2.62 \%$ & 0.13 & $23.08 \%$ & $1.05 \%$ & $2.62 \%$ \\
& 4 & $20.00 \%$ & $28.96 \%$ & $42.79 \%$ & 2.14 & $30.77 \%$ & $17.12 \%$ & $42.79 \%$ \\
& 5 & $20.00 \%$ & $24.82 \%$ & $36.68 \%$ & 1.83 & $23.08 \%$ & $14.67 \%$ & $36.68 \%$ \\
\hline \multirow{5}{*}{$\mathrm{MV}$} & 1 & $74.46 \%$ & $8.63 \%$ & $74.46 \%$ & 1.00 & $46.31 \%$ & $7.14 \%$ & $74.46 \%$ \\
& 2 & $0.00 \%$ & $13.81 \%$ & $0.00 \%$ & 1.60 & $0.00 \%$ & $11.43 \%$ & $0.00 \%$ \\
& 3 & $14.93 \%$ & $8.63 \%$ & $14.93 \%$ & 1.00 & $27.85 \%$ & $7.14 \%$ & $14.93 \%$ \\
& 4 & $9.71 \%$ & $8.63 \%$ & $9.71 \%$ & 1.00 & $24.16 \%$ & $7.14 \%$ & $9.71 \%$ \\
& 5 & $0.90 \%$ & $8.63 \%$ & $0.90 \%$ & 1.00 & $1.68 \%$ & $7.14 \%$ & $0.90 \%$ \\
\hline \multirow{5}{*}{$\mathrm{MDP}$} & 1 & $27.78 \%$ & $4.42 \%$ & $10.87 \%$ & 0.39 & $10.87 \%$ & $2.94 \%$ & $10.87 \%$ \\
& 2 & $13.89 \%$ & $8.85 \%$ & $10.87 \%$ & 0.78 & $10.87 \%$ & $5.88 \%$ & $10.87 \%$ \\
& 3 & $33.33 \%$ & $13.27 \%$ & $39.13 \%$ & 1.17 & $39.13 \%$ & $8.82 \%$ & $39.13 \%$ \\
& 4 & $25.00 \%$ & $17.69 \%$ & $39.13 \%$ & 1.57 & $39.13 \%$ & $11.76 \%$ & $39.13 \%$ \\
& 5 & $0.00 \%$ & $15.92 \%$ & $0.00 \%$ & 1.41 & $0.00 \%$ & $10.59 \%$ & $0.00 \%$ \\
\hline & 1 & $35.55 \%$ & $6.00 \%$ & $20.00 \%$ & 0.56 & $15.90 \%$ & $3.85 \%$ & $20.00 \%$ \\
& 2 & $17.77 \%$ & $12.00 \%$ & $20.00 \%$ & 1.13 & $15.90 \%$ & $7.69 \%$ & $20.00 \%$ \\
& 3 & $22.14 \%$ & $9.63 \%$ & $20.00 \%$ & 0.90 & $29.71 \%$ & $6.17 \%$ & $20.00 \%$ \\
& 4 & $12.47 \%$ & $17.11 \%$ & $20.00 \%$ & 1.60 & $22.30 \%$ & $10.97 \%$ & $20.00 \%$ \\
& 5 & $12.07 \%$ & $17.67 \%$ & $20.00 \%$ & 1.66 & $16.20 \%$ & $11.32 \%$ & $20.00 \%$ \\
\hline \hline
\end{tabular}

\subsection{Risk-based allocation}

In the first part of this section, we present the concept of risk parity funds. In the second part, we show how risk budgeting allocation could be used to build the strategic asset allocation of long-term investors.

\subsubsection{Risk parity funds}

The business of diversified funds has suffered a lot of criticisms, both from a theoretical and a practical point of view. If we consider the modern theory of portfolio management, mean-variance portfolios of risky assets define the set of efficient portfolios, that is the efficient frontier of Markowitz (see Figure 4). If we introduce a risk-free asset, the efficient frontier becomes a straight line called the security (or capital) market line. This frontier is a graphical representation of the risk-return profile of portfolios invested in the risk-free asset and a particular portfolio, also called the tangency portfolio. This last one is the portfolio which belongs to the efficient frontier and which maximises the Sharpe ratio. A consequence is that the portfolios of the security market line dominates the portfolios of the efficient frontier. The allocation choice between the cash and the tangency portfolio depends on the investor profile. Generally, we distinguish three profiles depending on the risk tolerance:

- Conservative (low risk tolerance)

- Moderate (medium risk tolerance)

- Agressive (high risk tolerance) 
Figure 4: The asset allocation puzzle
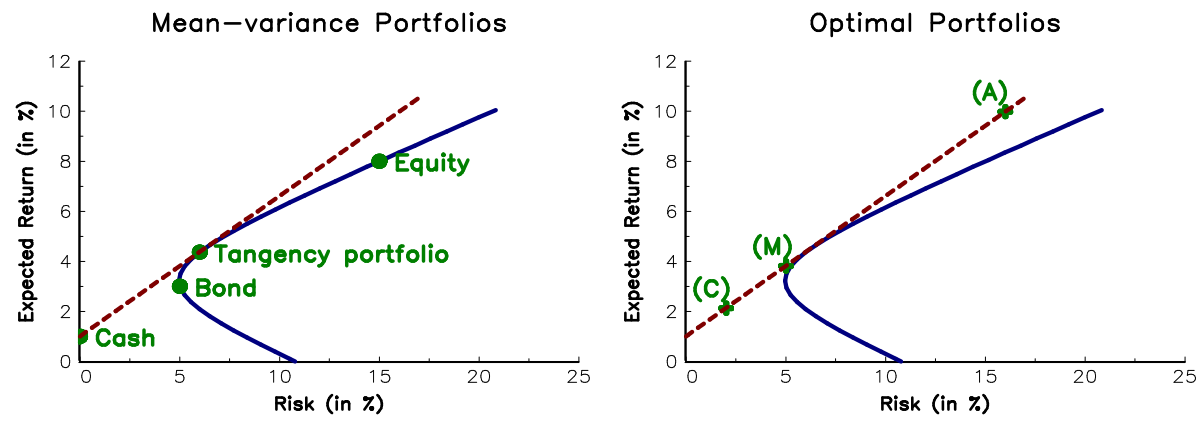

Diversified Funds

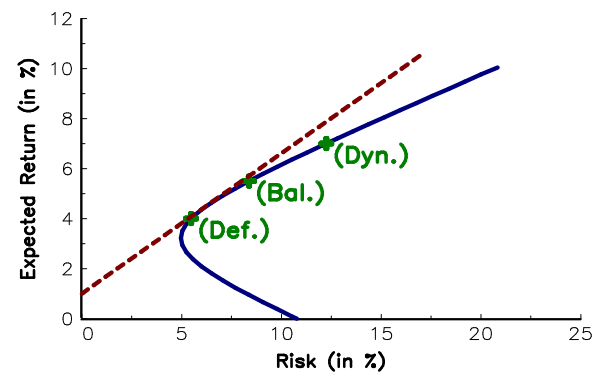

In Figure 4, these three profiles have been represented in the top-right quadrant. Because the conservative investor has a smaller appetite for risk than the aggressive investor, its portfolio will contain more cash and less risky assets. But the relative proportions between risky assets are the same for the conservative and agressive investors. In practice, it is inefficient to pay fees just to leverage or deleverage the tangency portfolio. However, the business of diversified funds is largely based on this framework. Generally, we distinguish three profiles depending on the benchmark of the diversified fund:

- Defensive ( $80 \%$ of bonds and $20 \%$ of equities)

- Balanced (50\% of bonds and $50 \%$ of equities)

- Dynamic (20\% of bonds and $80 \%$ of equities)

In this case, the distinction between the three fund profiles depends on the weight of equities of the corresponding benchmark. In Figure 4, these three profiles have been represented in the bottom quadrant. Contrary to the optimal portfolios corresponding to the three investor profiles, the portfolios corresponding to these three lifestyle funds are located on the efficient frontier and not on the security market line. The relationship between investor profiles and fund profiles is then not easy. The business of diversified funds suggests however that a defensive (resp. balanced or dynamic) fund profile matches the need of a conservative (resp. moderate or agressive) investor profile. But we clearly face a gap between the theory and the industry practice. This paradox is called in finance the asset allocation puzzle ${ }^{21}$. From the practical point of view, the main criticisms come from the fact that the allocation of diversified funds is very static and does not justify the high level of management fees. More

\footnotetext{
${ }^{21}$ Even if we could partially solve this problem (Bajeux-Besnainou et al., 2003), the controversy is still relevant today (Campbell and Viciera, 2002).
} 
Managing Risk Exposures using the Risk Budgeting Approach

recently, the industry has launched similar products called flexible funds in order to answer these criticisms.

In what follows, the bond and equity asset classes are represented by the Citigroup WGBI All Maturities Index and the MSCI World TR Net Index. In Figure 5, we have simulated the evolution of the risk contribution of these two asset classes for the three diversified funds with a one-year rolling empirical covariance matrix. We notice that these risk contributions are time-varying, especially for defensive and balanced funds. For defensive funds, the bond asset class has a larger risk contribution than the equity asset class. For balanced funds, we obtain the opposite, meaning that even if they are very well balanced in terms of weights, they are certainly not in terms of risk diversification. For dynamic funds, the risk is almost entirely explained by equities. In some sense, dynamic funds may be viewed as a deleverage of an equity exposure. We also notice that there is no mapping between fund profiles and volatility regimes. For example, the volatility of a dynamic fund in 2006 is smaller than the volatility of a balanced fund in 2009 .

Figure 5: Risk contributions of diversified funds
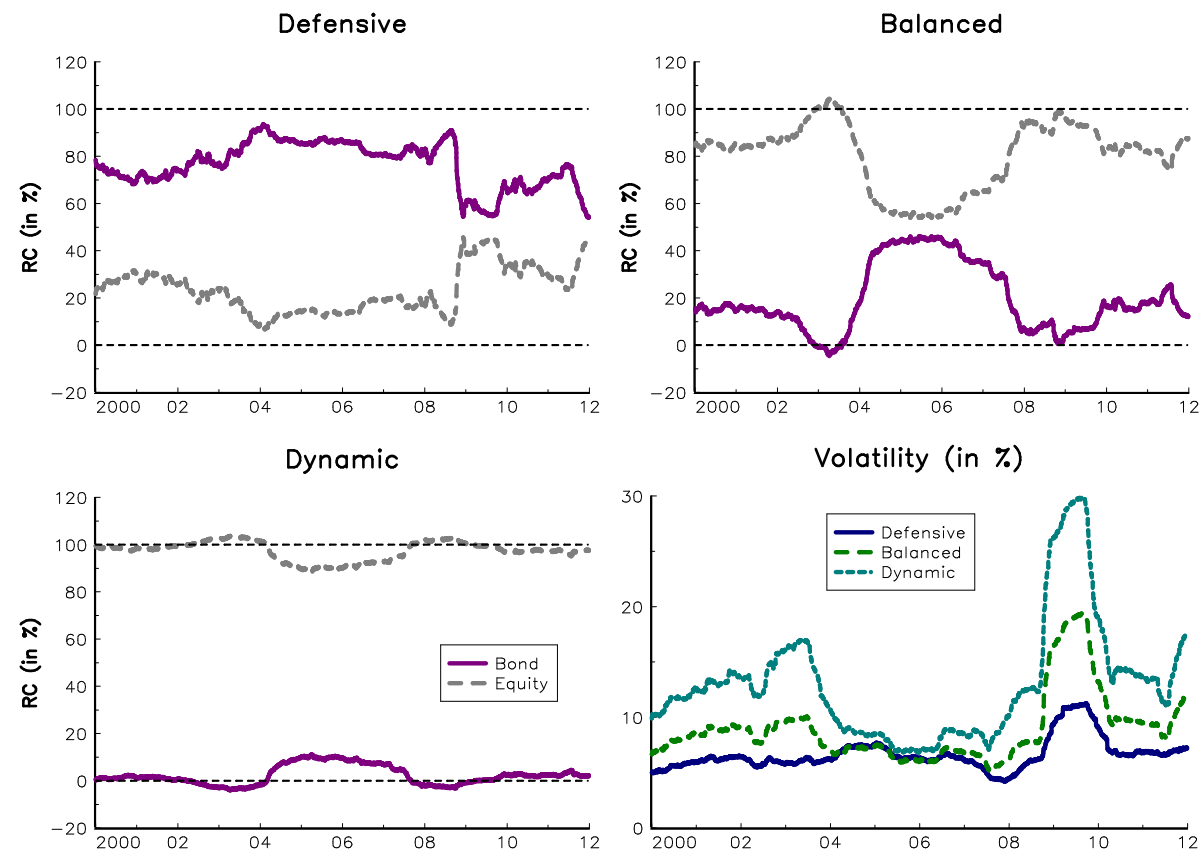

These drawbacks lead the investment industry to propose an alternative to these diversified funds. A risk parity (RP) fund is an ERC strategy on multi-assets classes:

"Diversify, but diversify by risk, not by dollars-that is, take a similar amount of risk in equities and in bonds" (Asness et al., 2012).

Applying this concept to our example is equivalent to build a portfolio where the risk of the bond asset class is equal to the risk of the equity asset class. If we assume that we rebalance the portfolio every month, we obtain the dynamic allocation given in Figure 6 . The simulated performance of this risk parity fund is reported in the bottom-right quadrant. It is difficult to compare it to those of the diversified funds (bottom-left quadrant), but we 
Figure 6: Comparison of diversified and risk parity funds

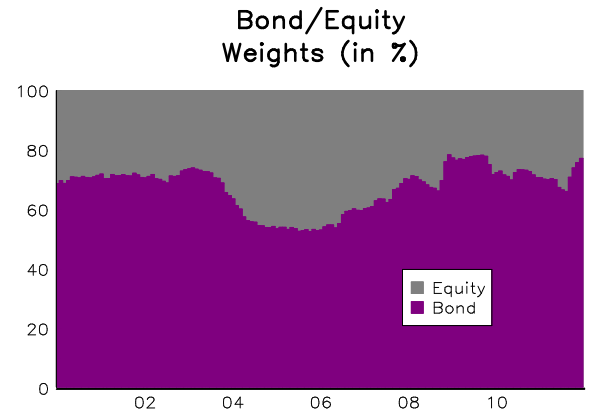

Performance of diversified funds

Performance of risk parity funds
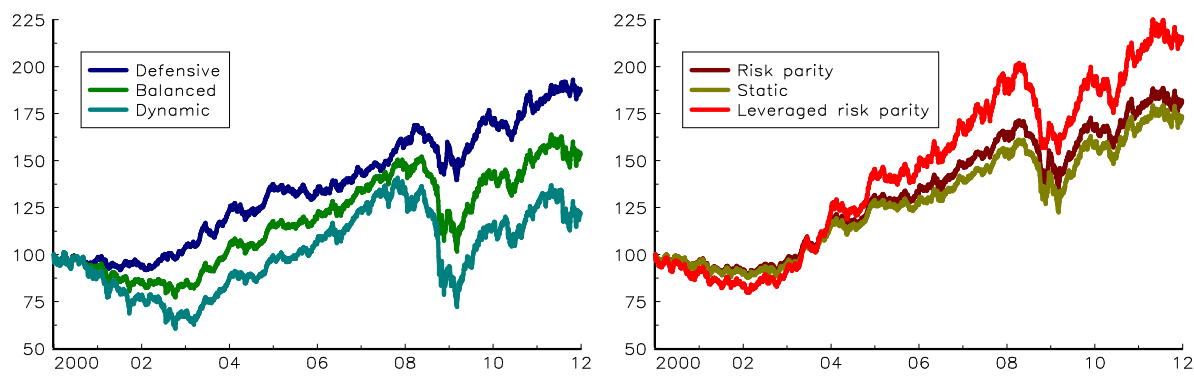

notice that risk parity and defensive funds are close. We have also reported the simulated performance when the weights are constant and are equal to the average weights of the risk parity fund. Compared to this static fund, the RP fund presents an outperformance of 40 bps and a volatility smaller than 35 bps. Sometimes, the ERC strategy is combined with a leverage effect in order to obtain a more risky profile. For example, if we apply a $10 \%$ volatility target, we obtain the performance of the leveraged risk parity fund (bottom-right quadrant). The leverage RP fund has a volatility similar to the balanced fund, but a better performance.

Remark 3 In practice, risk parity funds use a larger universe than the example presented here. It could be exposures on American, European, Japanese and Emerging Markets equities, large cap and small cap equities, American and European sovereign bonds, inflation-linked, corporate and high yield bonds, etc.

\subsubsection{Strategic asset allocation}

Strategic asset allocation (SAA) is the choice of equities, bonds, and alternative assets that the investor wishes to hold for the long-run, usually from 10 to 50 years. Combined with tactical asset allocation (TAA) and constraints on liabilities, it defines the long-term investment policy of pension funds. By construction, SAA requires long-term assumptions of asset risk/return characteristics as a key input. It could be done using macroeconomic models and forecasts of structural factors such as population growth, productivity and inflation (Eychenne et al., 2011). Using these inputs, one may obtain a SAA portfolio using a mean-variance optimization procedure. Because of the uncertainty of these inputs and the instability of mean-variance portfolios, a lot of institutional investors prefer to use these figures as a criteria to select the asset classes they would like to have in their strategic portfolio and to define the corresponding risk budgets. 
Managing Risk Exposures using the Risk Budgeting Approach

Let us illustrate this process with an example. We consider a universe of nine asset classes : US Bonds 10Y (1), EURO Bonds 10Y (2), Investment Grade Bonds (3), High Yield Bonds (4), US Equities (5), Euro Equities (6), Japan Equities (7), EM Equities (8) and Commodities (9). In Table 7, we indicate the long-run statistics used to compute the strategic asset allocation ${ }^{22}$. Based on these statistics and its constraints, the pension fund decides to define its strategic portfolio according to the risk budgets given in Figure 7 .

Table 7: Expected returns, risks and correlations (in \%)

\begin{tabular}{|c|c|c|c|c|c|c|c|c|c|c|c|}
\hline & $\mu_{i}$ & $\sigma_{i}$ & (1) & (2) & (3) & (4) & $\begin{array}{r}\rho_{i, j} \\
(5)\end{array}$ & (6) & (7) & (8) & (9) \\
\hline (1) & 4.2 & 5.0 & 100 & & & & I & & & & \\
\hline (2) & 3.8 & 5.0 & 80 & 100 & & & i & & & & \\
\hline (3) & 5.3 & 7.0 & 60 & 40 & 100 & & i & & & & \\
\hline (4) & 10.4 & 10.0 & -20 & -20 & 50 & 100 & & & & & \\
\hline$\overline{(5)}$ & $\overline{9} . \overline{2}$ & 15.0 & -10 & $-2 \overline{0}$ & 30 & 60 & $\overline{100}$ & & & & \\
\hline (6) & 8.6 & 15.0 & -20 & -10 & 20 & 60 & 90 & 100 & & & \\
\hline (7) & 5.3 & 15.0 & -20 & -20 & 20 & 50 & 70 & 60 & 100 & & \\
\hline (8) & 11.0 & 18.0 & -20 & -20 & 30 & 60 & 70 & 70 & 70 & 100 & \\
\hline$\overline{(9)}$ & $\overline{8} . \overline{8}$ & $\overline{3} 0.0$ & 0 & $\overline{0}$ & 10 & $\overline{2}$ & $2 \overline{0}$ & $\overline{2} 0$ & $3 \overline{0}$ & $\overline{3} 0$ & $10 \overline{0}$ \\
\hline
\end{tabular}

Figure 7: Risk budgeting policy of the pension fund

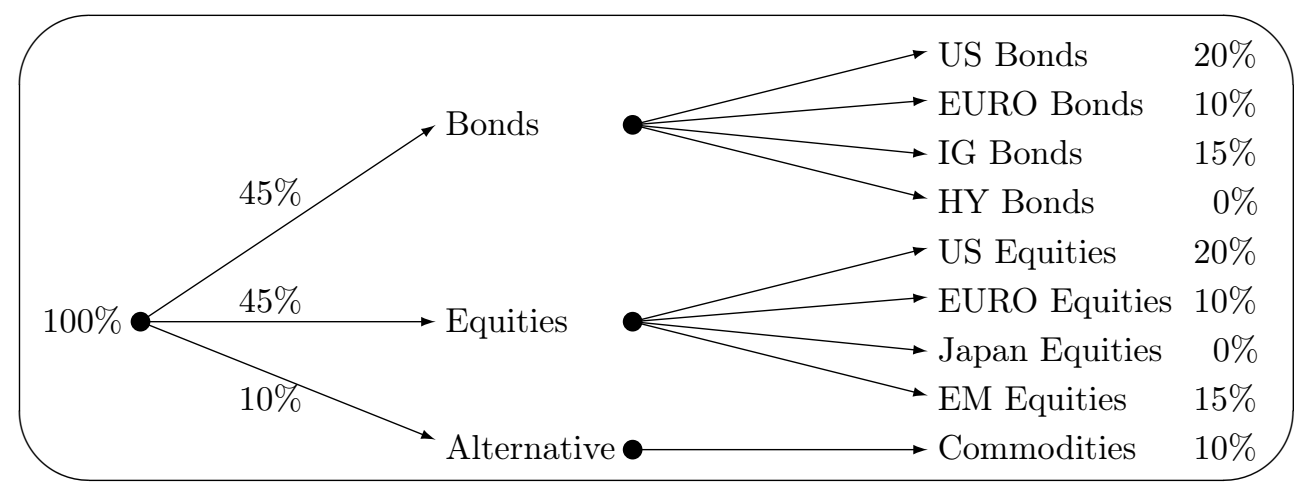

If we match these risk budgets, we obtain the solution RB given in Table 8. Of course, the pension fund may modify this strategic portfolio by using the expected returns. It could be done in Black-Litterman or tracking error frameworks. For example, if we would like to maximize the expected return of the portfolio according to a $1 \%$ tracking error with respect to the RB portfolio, we obtain the $\mathrm{RB}^{\star}$ portfolio given in Table 8. We could compare this modified portfolio with the mean-variance optimized (MVO) portfolio which has the same ex-ante volatility. Results are reported in Table 8 and in Figure 8. First, we notice that the two portfolios $\mathrm{RB}^{\star}$ and MVO are very close in terms of risk/return profile. Second, the $\mathrm{RB}^{\star}$ portfolio is much more diversified than the MVO portfolio, which concentrates $50 \%$ of its risk in the EM Equities asset class. The MVO portfolio is too far from the pension fund objective in terms of risk budgeting to be an acceptable strategic portfolio.

\footnotetext{
${ }^{22}$ This figures are taken from Eychenne et al. (2011) and Eychenne and Roncalli (2011)
} 
Figure 8: Efficient frontier

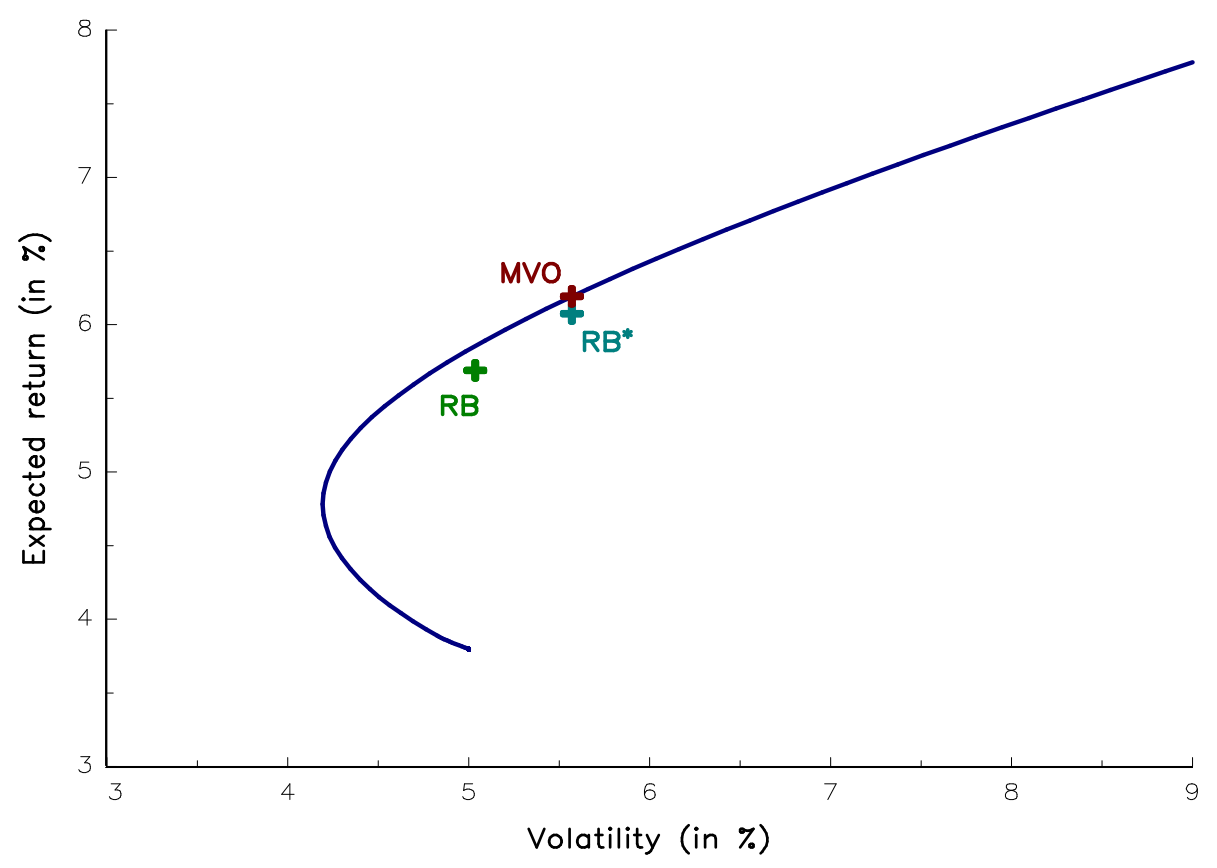

Table 8: Long-term strategic portfolios

\begin{tabular}{|c|c|c|c|c|c|c|}
\hline \multirow{2}{*}{ Asset class } & \multicolumn{2}{|c|}{ RB } & \multicolumn{2}{|c|}{$\mathrm{RB}^{\star}$} & \multicolumn{2}{|c|}{ MVO } \\
\hline & $x_{i}$ & $\mathrm{RC}_{i}$ & $x_{i}$ & $\mathrm{RC}_{i}$ & $x_{i}$ & $\mathrm{RC}_{i}$ \\
\hline (1) & $36.8 \%$ & $20.0 \%$ & $45.9 \%$ & $18.1 \%$ & $66.7 \%$ & $25.5 \%$ \\
\hline (2) & $21.8 \%$ & $10.0 \%$ & $8.3 \%$ & $2.4 \%$ & $0.0 \%$ & $0.0 \%$ \\
\hline (3) & $14.7 \%$ & $15.0 \%$ & $13.5 \%$ & $11.8 \%$ & $0.0 \%$ & $0.0 \%$ \\
\hline$(5)$ & $1 \overline{0} . \overline{2} \%$ & $\overline{2} 0 . \overline{0} \%$ & $\overline{10} 0.8 \%$ & $2 \overline{21.4 \%}$ & $\overline{7} . \overline{8} \%$ & $\overline{15} . \overline{1} \%$ \\
\hline (6) & $5.5 \%$ & $10.0 \%$ & $6.2 \%$ & $11.1 \%$ & $4.4 \%$ & $7.6 \%$ \\
\hline (8) & $7.0 \%$ & $15.0 \%$ & $11.0 \%$ & $24.9 \%$ & $19.7 \%$ & $49.2 \%$ \\
\hline$(9)$ & $\overline{3} . \overline{9} \%$ & $\overline{10.0 \%}$ & $4 . \overline{3} \%$ & $\overline{10} \overline{3} \%$ & $\overline{1} . \overline{5} \%$ & $\overline{2} . \overline{7} \%$ \\
\hline
\end{tabular}

\subsection{Risk-based indexation}

More recently, risk-budgeting techniques have been considered to build alternative benchmarks to market-cap indexes. These last ones have been particularly criticized by academics and market professionals and we observe a growing interest by sophisticated institutional investors. 


\subsubsection{Equity indexation}

Capitalization-weighted indexation is the most common way to gain access to broad equity market performance. It is often backed by results of modern portfolio theory because it is assimilated to the market portfolio. Moreover, it provides two main advantages: simplicity of management (low turnover and transaction costs) and ease of understanding and replication. However, it also presents some drawbacks. For example, capitalization-weighted indexation is by definition a trend-following strategy where momentum bias leads to bubble risk exposure as weights of best performers increase. Moreover, the absence of portfolio construction rules leads to concentration issues (in terms of sectors or stocks). In this context, the concept of alternative-weighted indexation emerged after the dot.com bubble. An alternativeweighted index is defined as an index in which assets are weighted differently than in the market capitalization approach. We generally distinguish two forms of alternative-weighted indexation: fundamental and risk-based. Fundamental indexation defines the weights as a function of economic metrics like dividends or earnings, whereas risk-based indexation defines the weights as a function of individual and common risks.

Figure 9: Performance of Eurostoxx 50 and ERC Eurozone indexes since January 1993

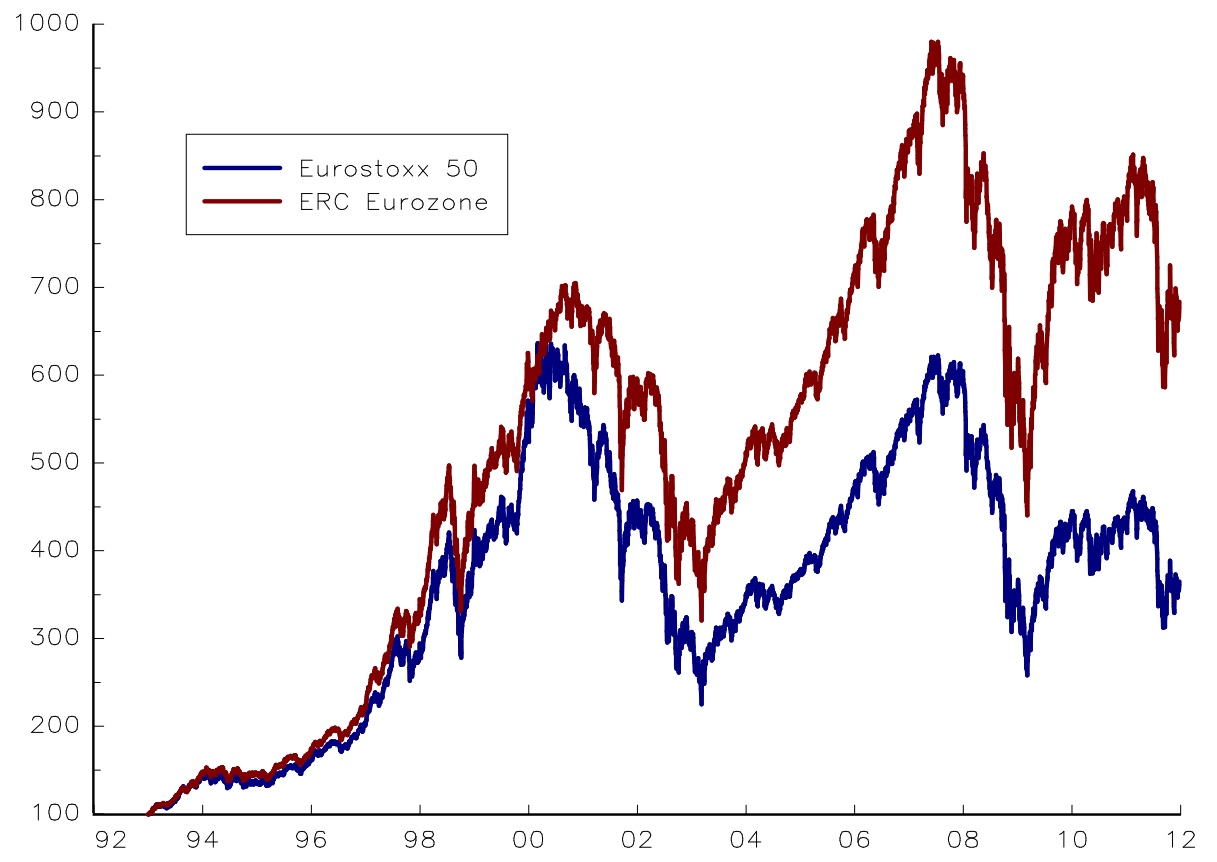

By construction, the ERC method belongs to the second form of alternative-weighted indexation. In Figure 9, we have reported the performance of the Eurostoxx $50 \mathrm{NR}$ index and the ERC Eurozone index ${ }^{23}$ from January 1993 to December 2011. The ERC Eurozone index has the same universe than the Eurostoxx $50 \mathrm{NR}$ index but it is rebalanced every month following the ERC method. We notice that the ERC Eurozone index has a smaller volatility $(21.2 \%$ versus $22.9 \%$ ) and a smaller drawdown $(55.1 \%$ versus $66.6 \%)$ than the Eurostoxx 50 index. What is more surprising is that the ERC Eurozone index outperforms the Eurostoxx 50 index (the yearly returns are respectively equal to $10.7 \%$ and $7.1 \%$ ). One way

\footnotetext{
${ }^{23}$ The corresponding Bloomberg tickers are respectively SX5T and SGIXERC.
} 
Managing Risk Exposures using the Risk Budgeting Approach

Table 9: Composition of Eurostoxx 50 and ERC Eurozone indexes (01/01/2012)

\begin{tabular}{|c|c|c|c|c|c|c|c|c|c|}
\hline \multirow[t]{2}{*}{ Name } & \multicolumn{4}{|c|}{ Eurostoxx 50} & \multicolumn{5}{|c|}{ ERC Eurozone } \\
\hline & $x_{i}$ & $\mathrm{RC}_{i}$ & $\tilde{\mu}_{i}$ & $\mathrm{PC}_{i}$ & $x_{i}$ & $\mathrm{RC}_{i}$ & $\tilde{\mu}_{i}$ & $\mathrm{PC}_{i}$ & $\mathrm{PC}_{i}^{\star}$ \\
\hline TOTAL SA & 6.55 & 5.00 & 5.74 & 5.00 & 2.45 & 2.00 & 5.72 & 2.00 & 2.02 \\
\hline SANOFI & 5.22 & 3.82 & 5.51 & 3.82 & 2.55 & 2.00 & 5.52 & 2.00 & 2.01 \\
\hline SIEMENS AG & 4.77 & 4.24 & 6.68 & 4.24 & 2.11 & 2.00 & 6.65 & 2.00 & 2.02 \\
\hline TELEFONICA SA & 3.98 & 3.46 & 6.55 & 3.46 & 2.16 & 2.00 & 6.52 & 2.00 & 2.02 \\
\hline BANCO SANTANDER SA & 3.76 & 4.37 & 8.76 & 4.37 & 1.64 & 2.00 & 8.58 & 2.00 & 2.06 \\
\hline BASF SE & 3.70 & 3.91 & 7.96 & 3.91 & 1.78 & 2.00 & 7.90 & 2.00 & 2.03 \\
\hline ENI SPA & 3.17 & 2.82 & 6.71 & 2.82 & 2.09 & 2.00 & 6.69 & 2.00 & 2.01 \\
\hline UNILEVER NV & 3.08 & 1.00 & 2.43 & 1.00 & 5.55 & 2.00 & 2.53 & 2.00 & 1.94 \\
\hline BAYER AG & 3.05 & 2.91 & 7.17 & 2.91 & 1.97 & 2.00 & 7.12 & 2.00 & 2.02 \\
\hline SAP AG & 2.82 & 1.81 & 4.82 & 1.81 & 2.89 & 2.00 & 4.86 & 2.00 & 1.99 \\
\hline ANHEUSER-BUSCH INB & 2.63 & 1.21 & 3.46 & 1.21 & 3.95 & 2.00 & 3.55 & 2.00 & 1.96 \\
\hline ALLIANZ SE & 2.50 & 3.46 & 10.41 & 3.46 & 1.36 & 2.00 & 10.32 & 2.00 & 2.03 \\
\hline E.ON AG & 2.49 & 2.90 & 8.76 & 2.90 & 1.61 & 2.00 & 8.71 & 2.00 & 2.02 \\
\hline BBVA & 2.41 & 3.14 & 9.82 & 3.14 & 1.46 & 2.00 & 9.64 & 2.00 & 2.05 \\
\hline BNP PARIBAS & 2.26 & 4.00 & 13.30 & 4.00 & 1.08 & 2.00 & 13.04 & 2.00 & 2.05 \\
\hline DAIMLER AG & 2.26 & 2.56 & 8.53 & 2.56 & 1.64 & 2.00 & 8.57 & 2.00 & 2.00 \\
\hline DANONE & 2.22 & 1.15 & 3.89 & 1.15 & 3.53 & 2.00 & 3.98 & 2.00 & 1.97 \\
\hline LVMH & 2.18 & 1.97 & 6.79 & 1.97 & 2.04 & 2.00 & 6.89 & 2.00 & 1.99 \\
\hline GDF SUEZ & 2.10 & 2.22 & 7.93 & 2.22 & 1.76 & 2.00 & 7.94 & 2.00 & 2.01 \\
\hline DEUTSCHE BANK AG & 2.05 & 3.19 & 11.71 & 3.19 & 1.21 & 2.00 & 11.56 & 2.00 & 2.04 \\
\hline AIR LIQUIDE SA & 2.03 & 1.43 & 5.29 & 1.43 & 2.63 & 2.00 & 5.34 & 2.00 & 1.99 \\
\hline DEUTSCHE TELEKOM AG & 1.97 & 1.41 & 5.38 & 1.41 & 2.58 & 2.00 & 5.44 & 2.00 & 1.99 \\
\hline FRANCE TELECOM SA & 1.73 & 1.32 & 5.73 & 1.32 & 2.44 & 2.00 & 5.75 & 2.00 & 2.00 \\
\hline SCHNEIDER ELECTRIC SA & 1.67 & 2.27 & 10.24 & 2.27 & 1.37 & 2.00 & 10.26 & 2.00 & 2.01 \\
\hline ING GROEP NV & 1.60 & 3.00 & 14.08 & 3.00 & 1.00 & 2.00 & 14.03 & 2.00 & 2.02 \\
\hline VIVENDI & 1.56 & 1.42 & 6.84 & 1.42 & 2.04 & 2.00 & 6.89 & 2.00 & 2.00 \\
\hline ENEL SPA & 1.50 & 1.46 & 7.32 & 1.46 & 1.92 & 2.00 & 7.33 & 2.00 & 2.01 \\
\hline REPSOL YPF SA & 1.45 & 1.48 & 7.68 & 1.48 & 1.84 & 2.00 & 7.64 & 2.00 & 2.02 \\
\hline L'OREAL & 1.44 & 0.82 & 4.30 & 0.82 & 3.20 & 2.00 & 4.38 & 2.00 & 1.97 \\
\hline AXA SA & 1.39 & 2.43 & 13.13 & 2.43 & 1.08 & 2.00 & 13.03 & 2.00 & 2.03 \\
\hline INTESA SANPAOLO & 1.35 & 2.48 & 13.82 & 2.48 & 1.03 & 2.00 & 13.64 & 2.00 & 2.04 \\
\hline VINCI SA & 1.34 & 1.45 & 8.16 & 1.45 & 1.71 & 2.00 & 8.19 & 2.00 & 2.00 \\
\hline IBERDROLA SA & 1.32 & 1.28 & 7.30 & 1.28 & 1.93 & 2.00 & 7.27 & 2.00 & 2.02 \\
\hline VOLKSWAGEN AG & 1.24 & 1.45 & 8.79 & 1.45 & 1.57 & 2.00 & 8.91 & 2.00 & 1.98 \\
\hline BMW AG & 1.24 & 1.33 & 8.05 & 1.33 & 1.72 & 2.00 & 8.17 & 2.00 & 1.99 \\
\hline ASSICURAZIONI GENERALI & 1.16 & 1.18 & 7.68 & 1.18 & 1.84 & 2.00 & 7.63 & 2.00 & 2.02 \\
\hline PHILIPS ELEC(KON) & 1.14 & 1.08 & 7.09 & 1.08 & 1.97 & 2.00 & 7.14 & 2.00 & 2.00 \\
\hline MUENCHENER RUECKVER & 1.14 & 1.08 & 7.14 & 1.08 & 1.96 & 2.00 & 7.15 & 2.00 & 2.01 \\
\hline NOKIA OYJ & 1.06 & 1.01 & 7.21 & 1.01 & 1.89 & 2.00 & 7.42 & 2.00 & 1.95 \\
\hline INDITEX & 1.00 & 0.52 & 3.93 & 0.52 & 3.44 & 2.00 & 4.09 & 2.00 & 1.94 \\
\hline SOCIETE GENERALE & 1.00 & 2.06 & 15.55 & 2.06 & 0.92 & 2.00 & 15.29 & 2.00 & 2.05 \\
\hline RWE AG & 0.98 & 1.20 & 9.23 & 1.20 & 1.52 & 2.00 & 9.22 & 2.00 & 2.02 \\
\hline ARCELORMITTAL & 0.98 & 1.43 & 11.02 & 1.43 & 1.27 & 2.00 & 11.03 & 2.00 & 2.01 \\
\hline CIE DE SAINT-GOBAIN & 0.96 & 1.29 & 10.13 & 1.29 & 1.38 & 2.00 & 10.19 & 2.00 & 2.00 \\
\hline UNIBAIL-RODAMCO SE & 0.95 & 0.75 & 5.96 & 0.75 & 2.32 & 2.00 & 6.03 & 2.00 & 1.99 \\
\hline CRH PLC & 0.83 & 1.00 & 9.01 & 1.00 & 1.54 & 2.00 & 9.11 & 2.00 & 1.99 \\
\hline UNICREDIT SPA & 0.79 & 1.35 & 12.80 & 1.35 & 1.11 & 2.00 & 12.62 & 2.00 & 2.04 \\
\hline CARREFOUR SA & 0.75 & 0.84 & 8.43 & 0.84 & 1.65 & 2.00 & 8.50 & 2.00 & 1.99 \\
\hline TELECOM ITALIA SPA & 0.64 & 0.57 & 6.64 & 0.57 & 2.10 & 2.00 & 6.70 & 2.00 & 2.00 \\
\hline DEUTSCHE BOERSE AG & 0.56 & 0.46 & 5.17 & 0.46 & 2.22 & 2.00 & 5.30 & 2.00 & 1.65 \\
\hline
\end{tabular}


Figure 10: Performance contribution since January 1997

$P C_{i}-C W$
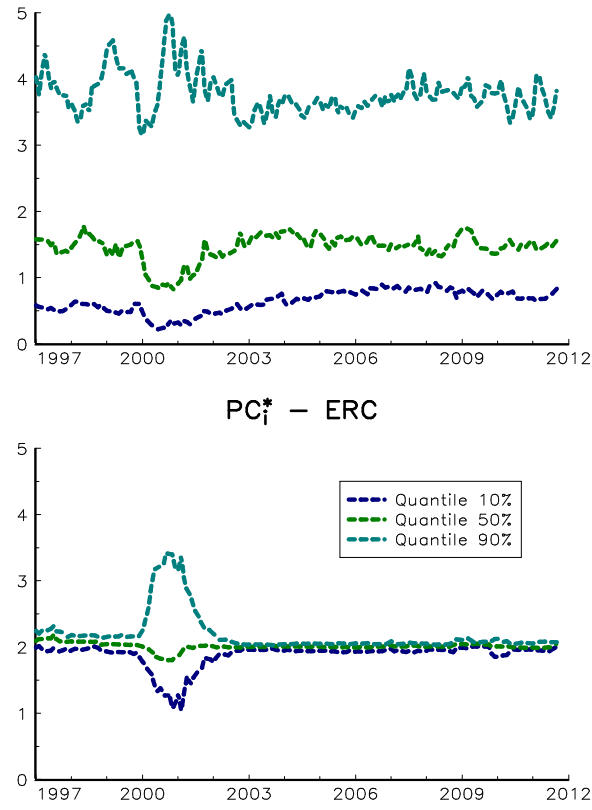

$P C_{i}-E R C$
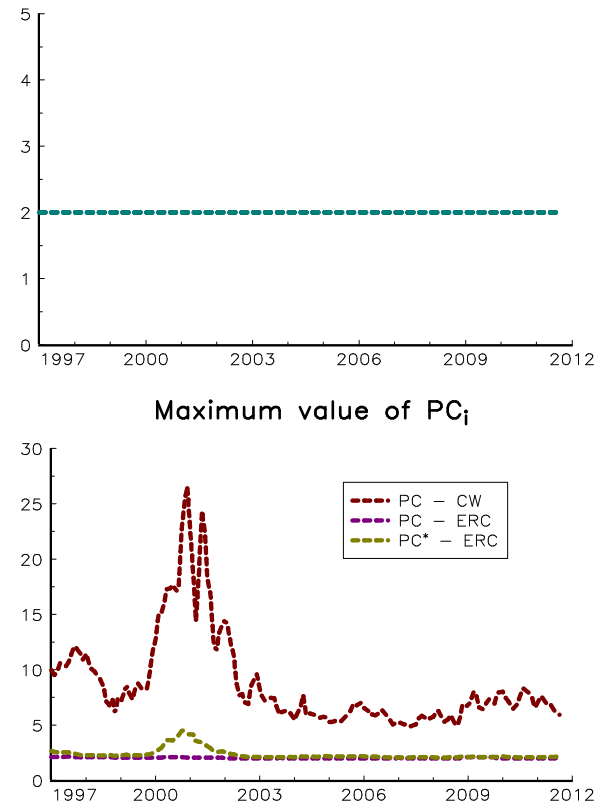

to explain this result is to remind that the ERC is the optimal portfolio whose performance contributions of assets are the same. In Table 9, we have reported the composition of the two indexes as at January 2012. We have also indicated the risk contribution $\mathrm{RC}_{i}$, the market price of expected return $\tilde{\mu}_{i}$ and the performance contribution ${ }^{24} \mathrm{PC}_{i} . \mathrm{PC}_{i}^{\star}$ corresponds to the performance contribution of the asset $i$ for the ERC portfolio when we consider the market price of the capitalization-weighted index. We verify that the ERC portfolio produces a more balanced portfolio in terms of performance contributions, even if we use the measure $\mathrm{PC}_{i}^{\star}$ instead of $\mathrm{PC}_{i}$. In Figure 10, we have reported the 10th and 90th percentiles and the maximum value taken by the performance contribution from January 1997 to December 2011. We observe that the capitalized-weighted (CW) Eurostoxx 50 index may concentrate ex-ante performance contribution in some stocks. This is not the case of the ERC index. In a certain way, the ERC index is a more passive strategy than the capitalized-weighted index, because it does not take any bets in terms of risk and performance contributions.

\subsubsection{Bond indexation}

Bond indexes face the same kind of problem than equity indexes. The weights are generally defined by the notional amount of debt, implying that asset management industry is dominated by the debt-weighted indexation. In the case of the sovereign bonds market, this means that each country in the index is given a weight proportional to its level of outstanding debt. As noted by Bruder et al. (2011), this method has a big advantage but faces some paradox:

"The simplicity of this approach and the recognition of a capitalisation-weighted index as the market portfolio has contributed to the success of the methodology.

\footnotetext{
${ }^{24}$ It is calibrated such that the average market prices of expected return is equal to $8 \%$.
} 
Yet, intuitively, it is easy to note a basic flaw in this allocation scheme, since it gives higher index weightings to the most indebted countries, regardless of their capacity to service their debt. A country facing financial hardship and trapped in a debt spiral to remain solvent would see its index weight increase until the whole mechanism collapses and an exclusion from the index occurs. Depending on the index, exclusion can be triggered by specific events, such as a downgrade or, in the worst case, a default."

Let $\operatorname{DEBT}_{i}$ and $\mathrm{GDP}_{i}$ be the amount of debt and the gdp of the country $i$. Bruder et al. (2011) compare four indexation methods:

- Debt weighting

$$
x_{i}=\frac{\mathrm{DEBT}_{i}}{\sum_{i=1}^{n} \mathrm{DEBT}_{i}}
$$

- Alternative weighting

- Fundamental indexation

A simple rule is to consider that the capacity of a country to service its debt is related to its gdp:

$$
x_{i}=\frac{\mathrm{GDP}_{i}}{\sum_{i=1}^{n} \mathrm{GDP}_{i}}
$$

- Risk-based indexation

The risk budgets may be proportional to the debt or to the gdp of the country:

$$
b_{i}=\frac{\mathrm{DEBT}_{i}}{\sum_{i=1}^{n} \mathrm{DEBT}_{i}} \text { or } b_{i}=\frac{\mathrm{GDP}_{i}}{\sum_{i=1}^{n} \mathrm{GDP}_{i}}
$$

One of the difficulty of risk-based indexation is to define the appropriate risk measure. Because the goal is to manage the sovereign credit risk, the authors propose to consider the volatility of the CDS basket which would perfectly hedge the credit risk of the bond portfolio. Using a SABR model (Hagan et al., 2002), they show that this risk measure depends on two "portfolio" parameters (the weight and the average duration) and three "market" parameters (the level and the volatility of the spread and the correlations between spreads).

In Figure 11, we have reported the evolution of the credit risk contributions of each country in the case of the Citigroup EGBI index. We notice the increase of Greece's risk contribution since 2008. On April 27th 2010, the country's sovereign debt rating was cut to BB + by Standard \& Poor's. Therefore Greece lost its investment grade status and exited the EGBI index at its next rebalancing date. The risk contribution of Greece reached a maximum of $28.5 \%$ on April 30th, 2010, while at the end of June 2010, just before its exit from the index, it remained very high, at $26 \%$. Since July 2010, we also notice an increase of the risk contribution of Portugal, Ireland, Italy and Spain. At the end of December 2011, Italy has a weight of $20.7 \%$ for a risk contribution of $40.3 \%$. In the same time, the weight and risk contribution of Germany are respectively $23.4 \%$ and $8 \%$.

Let us simulate the four indexing schemes by rebalancing the portfolio every month. Results are reported in Figure 12. We notice that weight budgeting indexes have a behavior different to risk budgeting indexes. These last ones offer a better performance, smaller volatility and drawdown. If we compare these indexation in terms of the sovereign credit risk measure (Figure 13), we obtain a similar behavior ${ }^{25}$ : the dynamics of the risk measure is different for weight budgeting indexes and risk budgeting indexes.

\footnotetext{
${ }^{25}$ We verify also that the volatility of the risk budgeting portfolio is smaller than the volatility of the weight budgeting portfolio.
} 
Figure 11: Evolution of risk contributions for the EGBI index

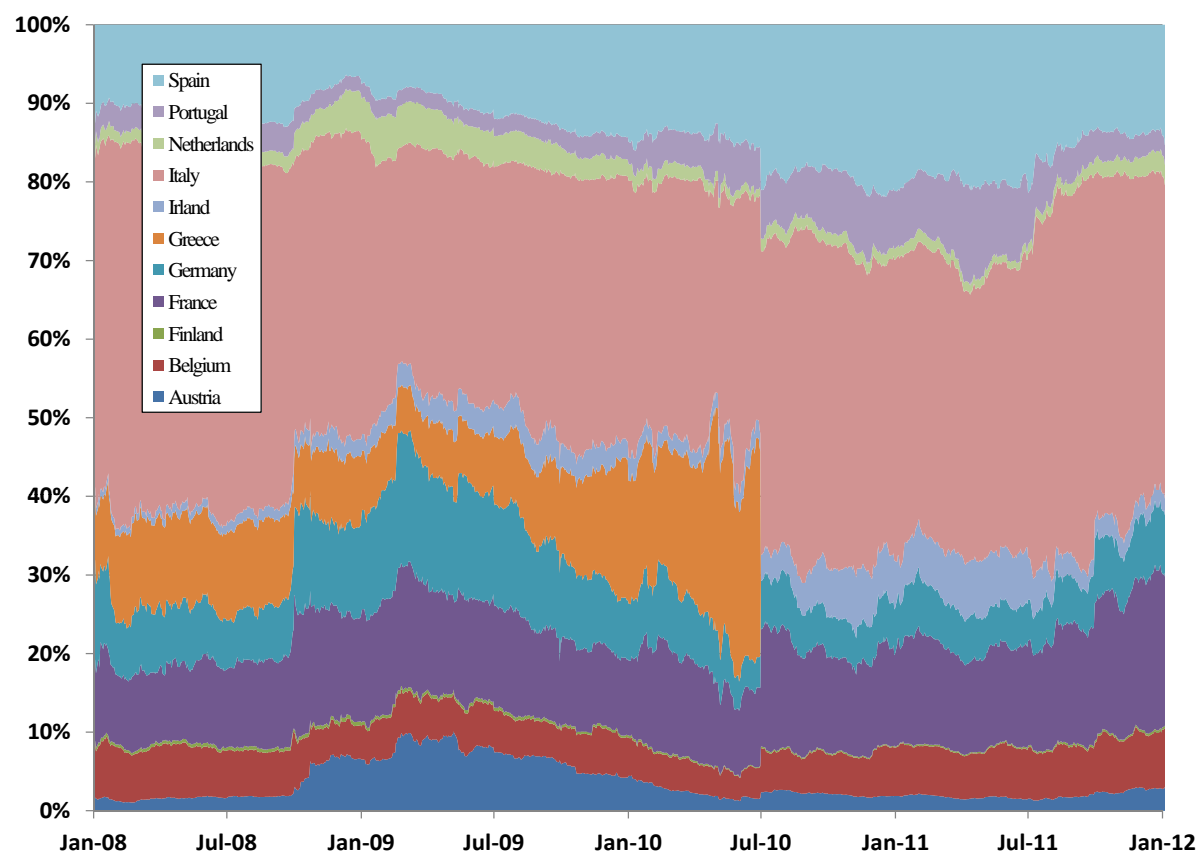

Figure 12: Simulated performance of the four indexing schemes

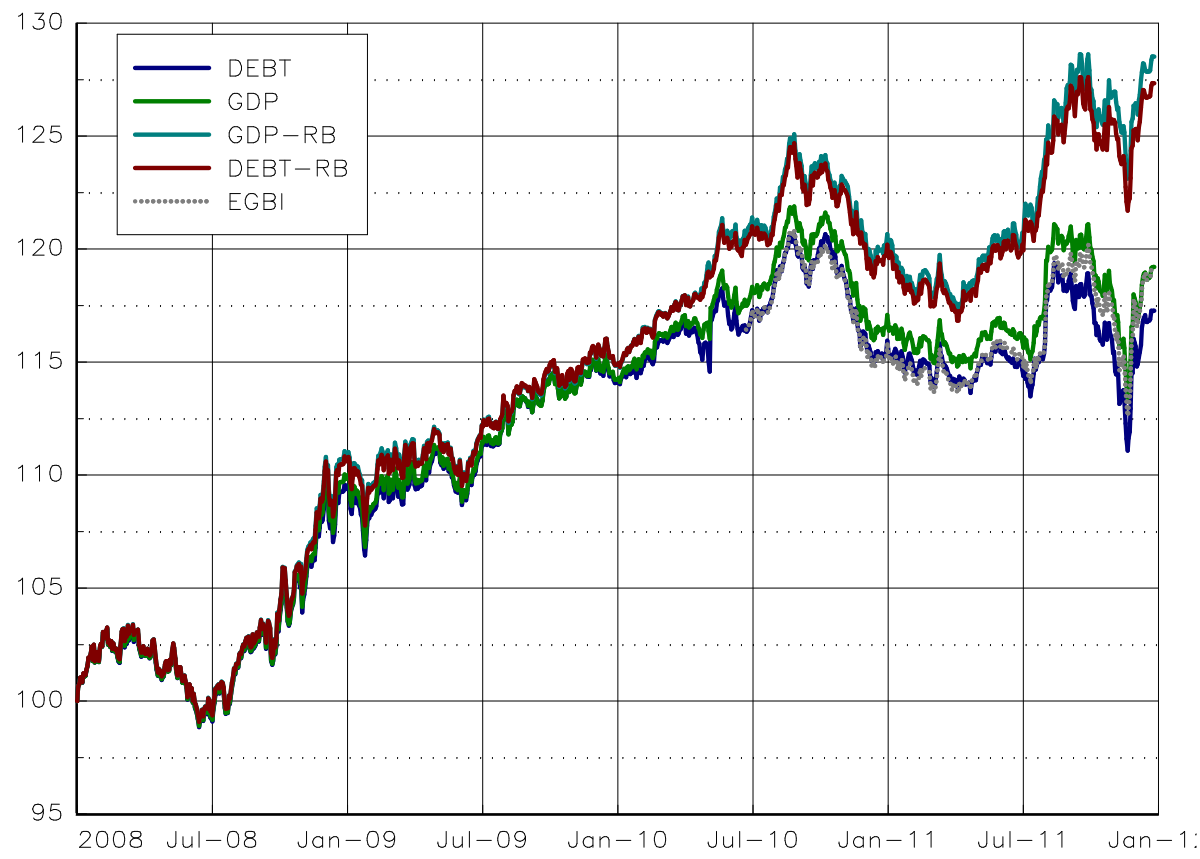


Figure 13: Ex-ante risk measure of the four indexing schemes

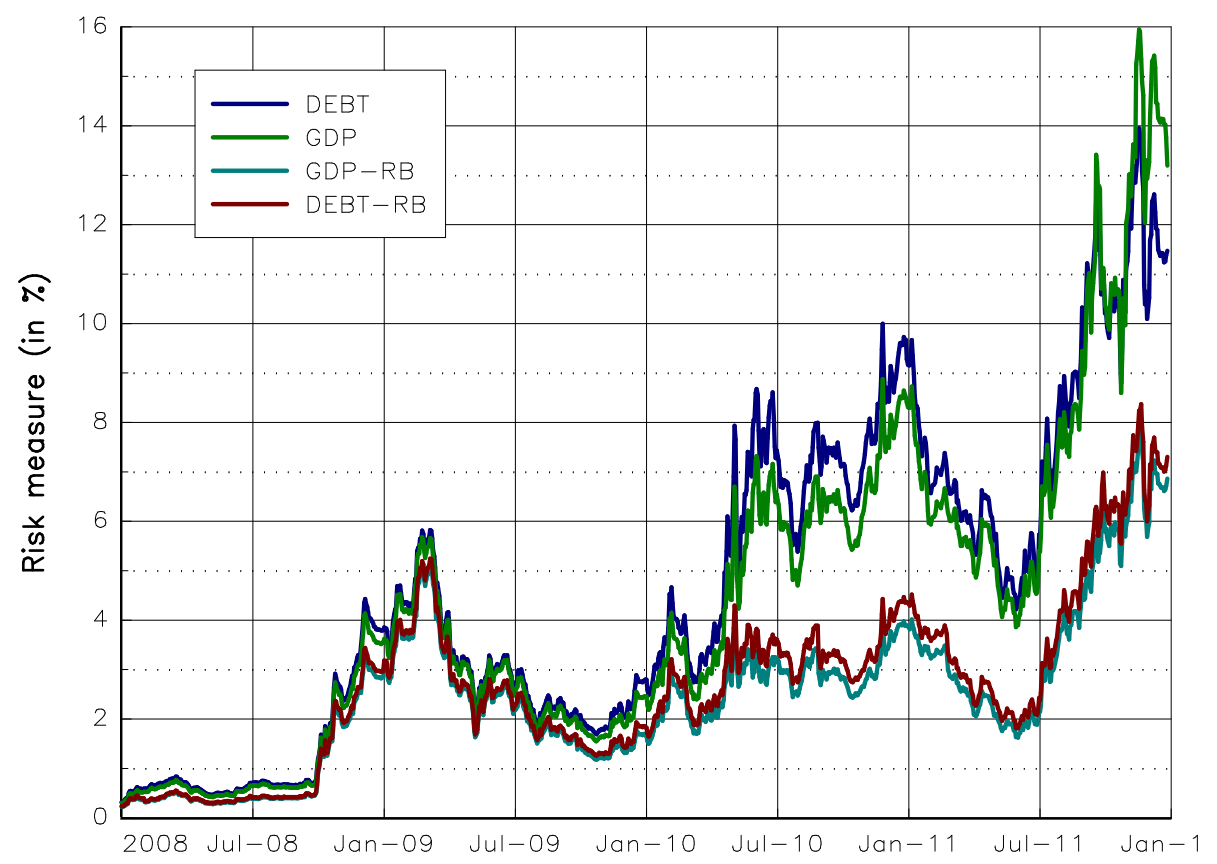

\section{Conclusion}

In this article, we study the risk budgeting approach for portfolio management. Risk budgeting portfolio is a generalization of the ERC portfolio presented in Maillard et al. (2010) when the risk budgets are not necessarily the same. It is also an heuristic method like equally-weighted or minimum-variance portfolios, because there is no financial theory that promotes the RB portfolio as an optimal portfolio. However, RB portfolios have been used by market practitioners for a long time. The success of the risk budgeting approach may certainly be explained by the risk management aspect of the methodology. It is particular true with the recent crisis.

Unfortunately, despite the increasing use of risk budgeting techniques, they are few results about the theoretical behavior of such portfolios. The objective of this paper was to understand this method in a deeper way. Several results have been obtained. First, we have completely defined the solution in the two-asset case and have partially characterized the solution in the general case. Second, we have shown that the volatility of the risk budgeting portfolio is located between those of minimum variance and weight budgeting portfolios. Third, we have also characterized the existence and uniqueness of such a portfolio.

In this paper, we present four main applications of the risk budgeting approach. They concern risk parity funds, strategic asset allocation, equity indexes and sovereign bonds benchmarks. All these applications are supported by the asset management industry and some sophisticated institutional investors already manage their money using the risk budgeting approach. One of the issue with this methodology is to understand what type of performance we could expect. Simulations and backtests show that it could deliver better performance than other portfolio methods. But understanding what are the sources of performance of the risk budgeting approach remains an open question. 
Managing Risk Exposures using the Risk Budgeting Approach

\section{References}

[1] Arnott, R., Hsu J. and Moore P. (2005), Fundamental indexation, Financial Analysts Journal, 61(2), pp. 83-99

[2] Artzner A., Delbaen F., Eber J.-M. and Heath D. (1999), Coherent Measures of Risk, Mathematical Finance, 9(3), pp. 203-228.

[3] Asness C.S., Frazzini A. and Pedersen L.H. (2012), Leverage Aversion and Risk Parity, Financial Analysts Journal, 68(1), pp. 47-59.

[4] Berkelaar A.B., Kobor A. and Tsumagari M. (2006), The Sense and Nonsense of Risk Budgeting, Financial Analysts Journal, 62(5), pp. 63-75.

[5] Bajeux-Besnainou I., Jordan J.V. and Portait R. (2003), Dynamic Asset Allocation for Stocks, Bonds, and Cash, Journal of Business, 76(2), pp. 263-287.

[6] Boотн D. and Fama E. (1992), Diversification and Asset Contributions, Financial Analyst Journal, 48(3), pp. 26-32.

[7] Boston Consulting Group (2011), Building on Success, Global Asset Management Report, www.bcg.com.

[8] Bruder B., Hereil P. and Roncalli T. (2011), Managing Sovereign Credit Risk, Journal of Indexes Europe, 1(4), pp. 20-27.

[9] Bruder B., Hereil P. and Roncalli T. (2011), Managing Sovereign Credit Risk in Bond Portfolios, Working Paper, www.ssrn.com/abstract $=1957050$.

[10] Campbell J.Y. and Viciera L.M. (2002), Strategic Asset Allocation, Oxford University Press.

[11] Choueifaty Y. and Coignard Y. (2008), Toward Maximum Diversification, Journal of Portfolio Management, 35(1), pp. 40-51.

[12] Clark G.L., Caerlewy-Smith E. and Marshall J.C. (2006), Pension Fund Trustee Competence: Decision Making in Problems Relevant to Investment Practice, Journal of Pension Economics and Finance, 5(1), pp. 91-110.

[13] Clarke R., De Silva H. and Thorley S. (2006), Minimum-variance portfolios in the U.S. equity market, Journal of Portfolio Management, 33(1), pp. 10-24.

[14] Demey P., Maillard S. and Roncalli T. (2010), Risk-Based Indexation, Lyxor White Paper Series, 1, www.lyxor.com.

[15] DeMiguel V., Garlappi L. and Uppal R. (2009), Optimal Versus Naive Diversification: How Inefficient is the 1/N Portfolio Strategy?, Review of Financial Studies, 22(5), pp. 1915-1953.

[16] Eychenne K., Martinetti S. and Roncalli T. (2011), Strategic Asset Allocation, Lyxor White Paper Series, 6, www.Iyxor.com.

[17] Eychenne K. and Roncalli T. (2011), Strategic Asset Allocation - An Update following the Sovereign Debt Crisis, Lyxor Short Paper, November, www.Iyxor.com. 
Managing Risk Exposures using the Risk Budgeting Approach

[18] Grinold R.C. and Kahn R.N. (2000), Active Portfolio Management: A Quantitative Approach for Providing Superior Returns and Controlling Risk, second edition, McGraw-Hill.

[19] Hagan P.S., Kumar D., Lesniewski A.S. and Woodward D.E. (2002), Managing Smile Risk, Wilmott Magazine, July, pp. 84-108.

[20] Hyvärinen A. and Oja E. (2000), Independent Component Analysis: Algorithms and Applications, Neural Networks, 13(4-5), pp. 411-430.

[21] Investment Company Institute (2011), Investment Company Fact Book - A Review of Trends and Activity in the Investment Company Industry, 51th edition, www.icifactbook. org.

[22] Jagannathan J. and Ma T. (2003), Reduction in Large Portfolios: Why Imposing the Wrong Constraints Helps, Journal of Finance, 58(4), pp. 1651-1683.

[23] Laloux L., Cizeau P., Bouchaud J-P. and Potters M. (1999), Noise Dressing of Financial Correlation Matrices, Physical Review Letters, 83(7), pp. 1467-1470.

[24] Ledoit, O. and Wolf, M. (2003), Improved Estimation of the Covariance Matrix of Stock Returns With an Application to Portfolio Selection, Journal of Empirical Finance, 10(5), pp. 603-621.

[25] Ledoit, O. and Wolf, M. (2004), Honey, I Shrunk the Sample Covariance Matrix, Journal of Portfolio Management, 30(4), pp. 110-119.

[26] Maillard S., Roncalli T. and Teiletche J. (2010), The Properties of Equally Weighted Risk Contributions Portfolios, Journal of Portfolio Management, 36(4), pp. $60-70$.

[27] Martellini L. (2008), Toward the Design of Better Equity Benchmarks, Journal of Portfolio Management, 34(4), pp. 1-8.

[28] Meucci A. (2005), Risk and Asset Allocation, Springer.

[29] Michaud R. (1989), The Markowitz Otimization Enigma: Is Optimized Optimal?, Financial Analysts Journal, 45(1), pp. 31-42.

[30] Qian E. (2006), On the Financial Interpretation of Risk Contributions: Risk Budgets Do Add Up, Journal of Investment Management, Fourth Quarter.

[31] Rahl L. (ed.) (2000), Risk Budgeting: A New Approach to Investing, Risk Books.

[32] Roncalli T. (2011), Understanding the Impact of Weights Constraints in Portfolio Theory, Working Paper, www.ssrn.com/abstract=1761625.

[33] Scherer B. (2007), Portfolio Construction \&3 Risk Budgeting, third edition, Risk Books.

[34] Scherer B. (2010), A New Look at Minimum Variance Investing, Working Paper, www. ssrn. com/abstract $=1681306$.

[35] Sharpe W.F. (2002), Budgeting and Monitoring Pension Fund Risk, Financial Analysts Journal, 58(5), pp. 74-86.

[36] TÜtÜncü R.H and Koenig M. (2004), Robust Asset Allocation, Annals of Operations Research, 132, pp. 132-157. 


\section{A Appendix}

\section{A.1 Solution of the risk budgeting problem when the constant cor- relation reaches its lower bound}

In the case of the constant correlation matrix, we have:

$$
x_{i} \sigma_{i}\left((1-\rho) x_{i} \sigma_{i}+\rho\left(\sum_{j=1}^{n} x_{j} \sigma_{j}\right)\right)=b_{i} \sigma^{2}(x)
$$

It comes that:

$$
(1-\rho) x_{i}^{2} \sigma_{i}^{2}+\frac{x_{i} \sigma_{i}}{x_{j} \sigma_{j}}\left(b_{j} \sigma^{2}(x)-(1-\rho) x_{j}^{2} \sigma_{j}^{2}\right)=b_{i} \sigma^{2}(x)
$$

If we assume that $\sigma(x)=0$, we obtain the following relationship:

$$
x_{i} \sigma_{i}=x_{j} \sigma_{j}=\varpi
$$

Let us compute the variance of the portfolio:

$$
\begin{aligned}
\sigma^{2}(x) & =\rho \sum_{i=1}^{n} x_{i} \sigma_{i}\left(\sum_{j=1}^{n} x_{j} \sigma_{j}\right)+(1-\rho) \sum_{i=1}^{n} x_{i}^{2} \sigma_{i}^{2} \\
& =\rho n^{2} \varpi^{2}+(1-\rho) n \varpi^{2}
\end{aligned}
$$

If $\rho=-1 /(n-1)$, we verify that the volatility of the portfolio is equal to zero. It proves that the ERC portfolio is the RB solution when the constant correlation reaches its lower bound.

\section{A.2 On the relationship between the optimization problem $(\mathbf{9})$ and the RB portfolio}

The Lagrangian function of the optimization problem (9) is:

$$
f\left(y ; \lambda, \lambda_{c}\right)=\sqrt{y^{\top} \Sigma y}-\lambda^{\top} y-\lambda_{c}\left(\sum_{i=1}^{n} b_{i} \ln y_{i}-c\right)
$$

The solution $y^{\star}$ verifies the following first-order condition:

$$
\frac{\partial f\left(y ; \lambda, \lambda_{c}\right)}{\partial y_{i}}=\frac{\partial \sigma(y)}{\partial y_{i}}-\lambda_{i}-\lambda_{c} \frac{b_{i}}{y_{i}}=0
$$

and the Kuhn-Tucker conditions:

$$
\left\{\begin{array}{l}
\min \left(\lambda_{i}, y_{i}\right)=0 \\
\min \left(\lambda_{c}, \sum_{i=1}^{n} b_{i} \ln y_{i}-c\right)=0
\end{array}\right.
$$

Because $\ln y_{i}$ is not defined for $y_{i}=0$, it comes that $y_{i}>0$ and $\lambda_{i}=0$. We notice that the constraint $\sum_{i=1}^{n} b_{i} \ln y_{i}=c$ is necessarily reached (because the solution can not be $y^{\star}=\mathbf{0}$ ), then $\lambda_{c}>0$ and we have:

$$
y_{i} \frac{\partial \sigma(y)}{\partial y_{i}}=\lambda_{c} b_{i}
$$


Managing Risk Exposures using the Risk Budgeting Approach

We verify that risk contributions are proportional to the risk budgets. Moreover, we remark that we face a well know optimization problem (minimizing a quadratic function subject to lower convex bounds) which has a solution. We then deduce the RB portfolio by normalizing the solution $y^{\star}$ such that the sum of weights equals one. Notice that the solution $x^{\star}$ may be found directly from the optimization problem (10) by using a constant $c^{\star}=c-\ln \left(\sum_{i=1}^{n} y_{i}^{\star}\right)$ where $c$ is the constant used to find $y^{\star}$.

The previous analysis is valid because $b_{i}>0$. If one or several risk budgets are set to zero, the solution is modified as follows. Let $\mathcal{N}$ be the set of assets such that $b_{i}=0$. In this case, the lagrangian function becomes:

$$
f\left(y ; \lambda, \lambda_{c}\right)=\sqrt{y^{\top} \Sigma y}-\lambda^{\top} y-\lambda_{c}\left(\sum_{i \notin \mathcal{N}} b_{i} \ln y_{i}-c\right)
$$

The solution $y^{\star}$ verifies the following first-order conditions:

$$
\frac{\partial f\left(y ; \lambda, \lambda_{c}\right)}{\partial y_{i}}= \begin{cases}\partial_{y_{i}} \sigma(y)-\lambda_{i}-\lambda_{c} \frac{b_{i}}{y_{i}}=0 & \text { if } i \notin \mathcal{N} \\ \partial_{y_{i}} \sigma(y)-\lambda_{i}=0 & \text { if } i \in \mathcal{N}\end{cases}
$$

If $i \notin \mathcal{N}$, the previous analysis is valid and we verify that risk contributions are proportional to the risk budgets:

$$
y_{i} \frac{\partial \sigma(y)}{\partial y_{i}}=\lambda_{c} b_{i}
$$

If $i \in \mathcal{N}$, we have to distinguish two cases. If $y_{i}=0$, it means that $\lambda_{i}>0$ and $\partial_{y_{i}} \sigma(y)>0$. In the other case, if $y_{i}>0$, it means that $\lambda_{i}=0$ and $\partial_{y_{i}} \sigma(y)=0$. The solution $y_{i}=0$ or $y_{i}>0$ if $i \in \mathcal{N}$ will depend on the structure of the covariance matrix $\Sigma$.

\section{A.3 On the relationship between $\sigma_{\mathrm{rb}}, \sigma_{\mathrm{wb}}$ and $\sigma_{\mathrm{mv}}$}

Let us start with the optimization problem (10) considered in the body part of the text:

$$
\begin{aligned}
x^{\star}(c)= & \arg \min \sqrt{x^{\top} \Sigma x} \\
\text { u.c. } & \left\{\begin{array}{l}
\sum_{i=1}^{n} b_{i} \ln x_{i} \geq c \\
\mathbf{1}^{\top} x=1 \\
\mathbf{0} \leq x \leq \mathbf{1}
\end{array}\right.
\end{aligned}
$$

We remark that if $c_{1} \leq c_{2}$, we have $\sigma\left(x^{\star}\left(c_{1}\right)\right) \leq \sigma\left(x^{\star}\left(c_{2}\right)\right)$ because the constraint $\sum_{i=1}^{n} b_{i} \ln x_{i}-$ $c \geq 0$ is less restrictive with $c_{1}$ than with $c_{2}$. We notice that if $c=-\infty$, the optimization problem is exactly the minimum variance problem, and $x^{\star}(-\infty)$ is the minimum variance portfolio. We notice that the function $\sum_{i=1}^{n} b_{i} \ln x_{i}$ is bounded and we have $\sum_{i=1}^{n} b_{i} \ln x_{i} \leq \sum_{i=1}^{n} b_{i} \ln b_{i}$. The only solution for $c=\sum_{i=1}^{n} b_{i} \ln b_{i}$ is $x_{i}^{\star}=b_{i}$, that is the weight budgeting portfolio. It comes that the solution for the general problem with $c \in\left[-\infty, \sum_{i=1}^{n} b_{i} \ln b_{i}\right]$ satisfies:

$$
\sigma\left(x^{\star}(-\infty)\right) \leq \sigma\left(x^{\star}(c)\right) \leq \sigma\left(x^{\star}\left(\sum_{i=1}^{n} b_{i} \ln b_{i}\right)\right)
$$

or:

$$
\sigma_{\mathrm{mv}} \leq \sigma\left(x^{\star}(c)\right) \leq \sigma_{\mathrm{wb}}
$$

Using the result of Appendix A.2, it exists a constant $c^{\star}$ such that $x^{\star}\left(c^{\star}\right)$ is the RB portfolio. It proves that the inequality holds:

$$
\sigma_{\mathrm{mv}} \leq \sigma_{\mathrm{rb}} \leq \sigma_{\mathrm{wb}}
$$

